\title{
BEAUTY IS A BEAST, FROG IS A PRINCE: ASSORTATIVE MATCHING WITH NONTRANSFERABILITIES*
}

\author{
Patrick Legros ${ }^{\dagger}$ and Andrew F. Newman ${ }^{\ddagger}$
}

April 2002; revised: December 2006

\begin{abstract}
We present sufficient conditions for monotone matching in environments where utility is not fully transferable between partners. These conditions involve not only complementarity in types of the total payoff to a match, as in the transferable utility case, but also monotonicity in type of the degree of transferability between partners. We apply our conditions to study some models of risk sharing and incentive problems, deriving new results for predicted matching patterns in those contexts.

KEYWORDS: Assortative matching, nontransferable utility, risk sharing, intrahousehold allocation, principal-agent.

JEL Numbers: C78, D13, J41
\end{abstract}

${ }^{*}$ We thank Ken Binmore, Patrick Bolton, Maristella Botticini, Bengt Holmstrom, Hide Ichimura, Boyan Jovanovic, Eric Maskin, Meg Meyer, Nicola Pavoni, Wolfgang Pesendorfer, Ilya Segal, Lones Smith, a co-editor and three anonymous referees, as well as seminar participants at Berkeley, Bonn, BU, Caltech, Gerzensee, Harvard/MIT, Northwestern, Princeton, Stanford, Toulouse, UCL, UCSD, UCSB, and ULB, for useful comments and discussion.

${ }^{\dagger}$ ECARES, Université Libre de Bruxelles, Avenue F.D. Roosevelt 50, C.P. 114, 1050 Brussels, Belgium: plegros@ulb.ac.be, http://homepages.ulb.ac.be/ plegros, and CEPR. This author benefited from the financial support of the Communauté française de Belgique (projects ARC 98/03-221and ARC00/05252) and EU TMR Network contract $n^{\circ}$ FMRX-CT98-0203.

${ }^{\ddagger}$ Boston University, Department of Economics, 270 Bay State Road, Boston, MA 02215, USA : afnewman@bu.edu; and CEPR. This author acknowledges support as the Richard B. Fisher Member, Institute for Advanced Study, Princeton, and as a Peter B. Kenen Fellow, Department of Economics, Princeton University. 


\section{Introduction}

For the economist analyzing household behavior, firm formation, or the labor market, the characteristics of matched partners are paramount. The educational background of men and women who are married, the financial positions of firms that are merging, or the productivities of agents who are working together, all matter for understanding their respective markets. Matching patterns serve as direct evidence for theory, figure in the econometrics of selection effects, facilitate theoretical analysis, and are even treated as policy variables.

Much is known about characterizing matching in the special case of transferable utility (TU). For instance, if the function representing the total payoff to the match satisfies increasing (decreasing) differences in the partners' attributes, then there will always be positive (negative) assortative matching, whatever the distribution of types. Because they are distribution-free, results of this sort are very powerful and easy to apply.

But in many areas of economic analysis, the utility among individuals is not fully transferable ("non-transferable," or NTU). Partners may be risk averse with limited insurance possibilities; incentive or enforcement problems may restrict the way in which the joint output can be divided; or policy makers may impose rules about how output may be shared within relationships. As Becker (1973) pointed out long ago, rigidities that prevent partners from costlessly dividing the gains from a match may change the matching outcome, even if the level of output continues to satisfy monotone differences in type.

While interest in the issues represented by the non-transferable case is both longstanding and lively (see for instance Farrell and Scotchmer, 1988 on production in partnerships; Rosenzweig and Stark, 1989 on risk sharing in households; and more recently, Lazear, 2000 on incentive schemes for workers; Ackerberg and Botticini, 2002 on sharecropping; and Besley and Ghatak, 2005 on the organizational design of nonprofit firms), for the analyst seeking to characterize the equilibrium matching pattern in such settings, there is little theoretical guidance. 
The purpose of this paper is to offer some. We present sufficient conditions for assortative matching that are simple to express, intuitive to understand, and, we hope, tractable to apply. We illustrate their use with some examples that are of independent interest.

The class of models we consider are two-person matching games without search frictions in which the utility possibility frontier for any pair of agents is a strictly decreasing function. After introducing the model, we review the logic of the classical transferable utility results, which leads us to propose the "generalized difference conditions" (GDC) that suffice to guarantee monotone matching for any type distribution (Proposition 1).

We also present sufficient differential conditions for monotone matching (Corollary 1). In addition to being straightforward to verify, the differential conditions offer additional insight into the forces governing matching. In particular, they highlight the role not only of the complementarity in partners' types that figures in the TU case, but also of a complementarity between an agent's type and his partner's payoff that is the new feature in the NTU case. This second complementarity entails that the degree of transferability (i.e., slope of the frontier) be monotone in type. Even if the output satisfies increasing differences in types, failure of the type-payoff complementarity may overturn the predictions of the TU model and lead instead to negative assortative matching or some more complex and/or distribution-dependent pattern.

Two examples illustrate the application of our general results. The first is a marriage market model in which partners vary in risk attitude and must share risks within their households. Sorting effects in this kind of model are important considerations in the econometrics of marriage and migration (Rosenzweig and Stark, 1989). Using the GDC, we show that partners will sort negatively in their Arrow-Pratt degree of risk tolerance. The differential conditions are applied to the second example, a principalagent model in which agents vary in their initial wealth, and principals vary in the riskiness of their projects. Matching effects in such a model are of direct interest in some applications (e.g., Newman, 1999; Prendergast, 2002) and have been offered as explanations for some seemingly puzzling empirical results (Ackerberg and Botticini, 
2002). In this example, the type-transferability relationship alone is responsible for the predicted matching pattern, which goes in a (possibly) unexpected direction.

Finally, we discuss the strength of the GDC, pointing out that they are ordinal conditions (Proposition 2), which broadens the scope of applicability of the local conditions; that they are necessary if one seeks a distribution-free condition for assortative matching (Proposition 3); and their distinction from established conditions in the literature (Sections 6.3 and 7.3). Section 7 discusses extensions of our results to variations of the standard matching framework, and Section 8 concludes.

The next section delves further into the ideas underlying the general theoretical analysis by examining a very simple example inspired by the one in Becker (1973).

\section{An Illustrative Example}

Suppose there are two types of men, $p^{\prime}<p$, and two types of women, $a^{\prime}<a$. The total "output" they produce when matched, as a function of the partners' types, is described by the matrix

$$
\begin{aligned}
& a^{\prime} \quad a \\
& \begin{array}{lll}
p^{\prime} & 4 & 7
\end{array} \\
& \begin{array}{lll}
p & 7 & 9
\end{array}
\end{aligned}
$$

Since $9-7<7-4$, the joint payoff function satisfies decreasing differences (DD). If payoffs are fully transferable, then it is well known that decreasing differences implies that a stable outcome will always involve negative assortative matching (NAM): high types will match with low types. If to the contrary we had a positive match of the form $\left\langle p^{\prime}, a^{\prime}\right\rangle,\langle p, a\rangle$ with equilibrium payoffs $\left(\pi\left(p^{\prime}\right), \omega\left(a^{\prime}\right)\right)$ and $(\pi(p), \omega(a))$, then there would always be a pair of types that could do strictly better for themselves: $\omega\left(a^{\prime}\right)+$ $\omega(a)=\left(4-\pi\left(p^{\prime}\right)\right)+(9-\pi(p))<\left(7-\pi\left(p^{\prime}\right)\right)+(7-\pi(p))$; thus $\omega(a)<7-\pi\left(p^{\prime}\right)$ or $\omega\left(a^{\prime}\right)<7-\pi(p) ; a^{\prime}$ could offer $p$ (or $a^{\prime}$ could offer $p$ ) slightly more than his current payoff and still get more for herself, destabilizing the positive match. The negative matching outcome maximizes total output.

Suppose instead that utility is not perfectly transferable, and consider the extreme 
case in which any departure from equal sharing within the marriage is impossible. For instance, the payoff to the marriage could be generated by the joint consumption of a local public good. Thus each partner in $\langle p, a\rangle$ gets 4.5 , each in $\left\langle p, a^{\prime}\right\rangle$ gets 3.5, etc. Now the only stable outcome is positive assortative matching (PAM): each $p$ is better off matching with $a$ (4.5) than with $a^{\prime}(3.5)$, and thus the "power couple" blocks a negative assortative match. As Becker noted, with nontransferability, the match changes, and aggregate performance suffers as well.

Of course this extreme form of nontransferability does not represent many situations of economic interest. What happens in the broad intermediate range? Introduce a dose of transferability by supposing that some compensation, say through the return of favors, makes it possible to depart from equal sharing. Consider two simple cases. In the first, the high types are "difficult," while the low types are "easy": beauty is a beast, frog is a prince. That is, utility is perfectly transferable between $p^{\prime}$ and $a^{\prime}, p^{\prime}$ can transfer to $a$, but not vice versa, and $a^{\prime}$ can transfer to $p$ but not vice versa. In the second case, the high types are easy and the low types difficult. See Figure 2.1, which depicts the utility possibility frontiers between pairs of types, assuming feasible transfers are made starting from the equal sharing point.

In the first case, the degree of transferability is decreasing in type, and in particular is changing in the same direction as (marginal) productivity. The unique outcome is NAM in this case: if things were otherwise, a high type could promise a low type almost 2.5 , garnering a bit over 4.5 for itself, and the low type will be happy to accept the offer (the only way this could not happen is if both $a^{\prime}$ and $p^{\prime}$ were getting at least 2.5 , which is an impossibility).

In the second case, the degree of transferability is increasing in type, opposite the direction that productivity increases, and this opposition between productivity and transferability is enough to overturn the TU outcome. The easygoing high types now can get no more than 3.5 out of a mixed relationship, so they prefer a match with each other, wherein 4.5 would be available to each. ${ }^{1}$

\footnotetext{
${ }^{1}$ This begs the question of how much transferability is needed for NAM. We leave it to the reader to
} 

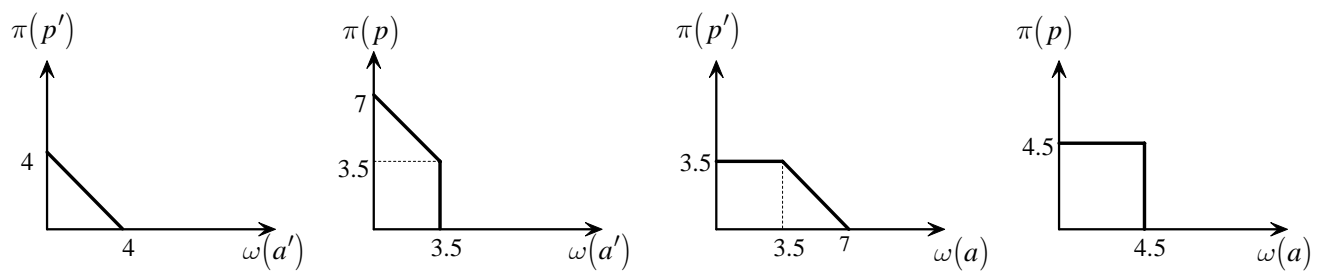

(1) Transferability decreasing in type
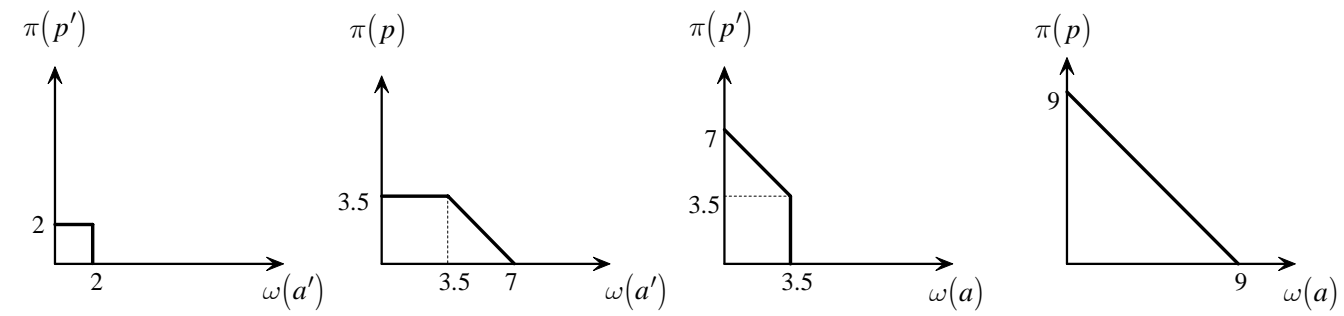

(2) Transferability increasing in type

Figure 2.1: Utility possibility frontiers

The basic intuitions contained in this example carry over to the general case, and are in a nutshell the content of our main results, Proposition 1 and Corollary $1 .^{2}$ Transferability, and its dependence on type, can be as important as productivity in determining the nature of sorting.

\section{The Model}

The economy is populated by a finite number of agents who differ in type, which is taken to be a real-valued attribute such as skill, wealth, or risk attitude. For expositional ease we focus on two-sided models, where agents are also distinguished by a binary "gender" (man-woman, firm-worker, etc.), but as we discuss in Section 7.2 the results can also be applied to "one-sided models." Payoffs exceeding that obtained in autarchy, which for

verify that if low types can transfer at a rate $\beta$, then there is $\beta^{*} \in(0,1)$ such that $\beta>\beta^{*}$ implies NAM and $\beta<\beta^{*}$ implies PAM.

${ }^{2}$ The only difference is that we shall require the frontiers to be strictly decreasing; the above examples could easily be modified to conform to this requirement without changing any conclusion. 
the general analysis we normalize to zero for all types ${ }^{3}$ are generated only if agents of opposite gender match.

Let $I$ be the set of agents on one side of the market and $J$ be the set of agents on the other. The description of a specific economy includes an assignment of individuals to types via maps $\rho: I \longrightarrow P$, and $\alpha: J \longrightarrow A$, where $P$ and $A$ are compact subsets of $\mathbb{R}$. To simplify the exposition we assume that $I$ and $J$ have the same cardinality. ${ }^{4}$

The object of analytical interest is the utility possibility frontier (since in equilibrium agents will always select an allocation on this frontier) for each possible pairing of agents. This frontier will be represented by a bounded continuous function $\phi: P \times A \times \mathbb{R}_{+} \rightarrow \mathbb{R}_{+}$; $\phi(p, a, v)$ denotes the maximum utility generated by a type $p \in P$ in a match with a type $a \in A$ who receives utility $v$. The maximum equilibrium payoff that $p$ could ever get in a match with $a$ is $\phi(p, a, 0)$, since $a$ would never accept a negative payoff. We assume throughout that $\phi(p, a, 0)$ is positive for all $(p, a)$ and that $\phi(p, a, v)$ is continuous and strictly decreasing in $v$ whenever $\phi(p, a, v)$ is positive. ${ }^{5}$

Let $\psi(a, p, \cdot)$ be the "quasi-inverse" of $\phi(p, a, \cdot): \psi(a, p, u)$ is the maximum payoff to a type- $a$ when his type- $p$ partner receives utility $u$. For any $p \in P, a \in A$, if $u \in$ $[0, \phi(p, a, 0)], \psi(a, p, u)$ solves $\phi(p, a, \psi(a, p, u))=u$, and if $u>\phi(p, a, 0), \psi(a, p, u)=0$.

\footnotetext{
${ }^{3}$ In many applications, the autarchy payoff varies with type. The analysis extends to this case almost without modification: see Section 7.1.

${ }^{4}$ This is basically without loss of generality; see Section 7.1 .

${ }^{5}$ While we take $\phi$ to be a primitive of the model for the general analysis, it will typically be derived from more fundamental assumptions about technology, preferences and choices made by the partners after they match, as in the examples in Section 5. The frontier can be generally expressed as

$$
\begin{gathered}
\phi(p, a, v)=\max _{x} U(x, p, a) \\
\text { s.t. } V(x, a, p) \geq v \\
x \in \Omega(p, a),
\end{gathered}
$$

where $U$ is the utility of the agent in $I, V$ the utility of the agent in $J, x$ the choice variables, and $\Omega(p, a) \subset \mathbb{R}^{n}$ is the choice set. The frontier will have the indicated properties if, for instance, $U$ and $V$ are continuous and locally nonsatiated, $\Omega(p, a)$ is continuous, and for each $(p, a), \Omega(p, a)$ has nonempty relative interior and contains a point $x_{p a}$ such that $U\left(x_{p a}, p, a\right)>0, V\left(x_{p a}, a, p\right)>0$.
} 
In general, of course, $\phi(p, a, w) \neq \psi(a, p, w)$.

We shall sometimes refer to the first argument of $\phi$ and $\psi$ as "own type," the second argument as "partner's type," and the third argument as "payoff."

The notation reflects two further assumptions of matching models, namely (1) that the payoff possibilities depend only on the types of the agents and not on their individual identities; and (2) the utility possibilities of the pair of agents do not depend on what other agents in the economy are doing, i.e., there are no externalities across coalitions.

The model encompasses the case of transferable utility (TU), in which there exists a production function $h(a, p)$ such that $\phi(p, a, v)$ can be written as $h(p, a)-v$ for $v \in[0, h(p, a)]$. In all other cases we have nontransferable utility (NTU).

Our concept of equilibrium is the core of the assignment game: it requires that agents in $I$ are matched to agents in $J$ in a stable way.

Definition 1. Payoffs $(u, v)$ are feasible for $(p, a) \in P \times A$ if $u \leq \phi(p, a, v)$ and $v \leq$ $\psi(a, p, 0)$.

Definition 2. An equilibrium specifies a one-to-one matching function $m: I \rightarrow J$ and payoff allocations $\pi^{*}: I \longrightarrow \mathbb{R}_{+}$and $\omega^{*}: J \longrightarrow \mathbb{R}_{+}$that satisfy the two following conditions.

(i) Feasibility of $\left(\pi^{*}, \omega^{*}\right)$ with respect to $m$ : for all $i \in I,\left(\pi^{*}(i), \omega^{*}(m(i))\right)$ is feasible for $(\rho(i), \alpha(m(i)))$.

(ii) Stability of $m$ with respect to $\left(\pi^{*}, \omega^{*}\right)$ : there do not exist $(i, j) \in I \times J$ and $v>\omega^{*}(j)$ such that $\phi(\rho(i), \alpha(j), v)>\pi^{*}(i)$.

Existence of equilibria in this class of economies has been established elsewhere (Kaneko, 1982).

Because $\phi(p, a, v)$ is strictly decreasing in $v$ on $(0, \psi(a, p, 0))$, an equilibrium satisfies equal treatment: if two agents with the same type had unequal equilibrium payoffs, it would be possible for the worse-treated to underbid the better-treated, contradicting stability. Thus, the equilibrium payoff functions $\pi^{*}$ and $\omega^{*}$ depend only on the individual's type, and it is enough to define type-dependent payoff functions $\pi: P \rightarrow \mathbb{R}_{+}$and 
$\omega: A \rightarrow \mathbb{R}_{+}$as $\pi(p)=\pi^{*}(\rho(i))$ when $\rho(i)=p$ for $i \in I$ and $\omega(a)=\omega^{*}(\alpha(j))$ when $\alpha(j)=a$ for $j \in J$.

Concepts of monotone matching are defined with respect to the matching correspondence between types. The matching function $m: I \rightarrow J$ generates a matching correspondence on types $\mathfrak{M}: P \rightrightarrows A$ :

$$
\mathfrak{M}(p)=\{\alpha(j), j \in J: \exists i \in I, \rho(i)=p \text { and } j=m(i)\}
$$

We say that $\mathfrak{M}$ is stable given $(\pi, \omega)$ if there do not exist $(p, a)$ and $v>\omega(a)$ such that $\phi(p, a, v)>\pi(p)$.

When $\mathfrak{M}$ is a monotone correspondence, matching is monotone. In two-sided models, there are only two types of monotone matching. An equilibrium displays positive assortative matching (PAM) if $p>p^{\prime}, a \in \mathfrak{M}(p), a^{\prime} \in \mathfrak{M}\left(p^{\prime}\right) \Longrightarrow a \geq a^{\prime}$. There is negative assortative matching (NAM) if $p>p^{\prime}, a \in \mathfrak{M}(p), a^{\prime} \in \mathfrak{M}\left(p^{\prime}\right) \Longrightarrow a^{\prime} \geq a$.

Say that an equilibrium is payoff equivalent to another if all agents have the same payoff in each equilibrium. We say an economy satisfies PAM (NAM) if each equilibrium is payoff equivalent to one in which the match satisfies PAM (NAM).

\section{Sufficient Conditions for Monotone Matching}

\subsection{Logic of the TU Case}

Recall the conventional transferable utility result, as that will provide guidance to the general case. In the TU case, only the total payoff $h(p, a)$ is relevant. The assumption that is often made about $h$ is that it satisfies increasing differences (ID): whenever $p>p^{\prime}$ and $a>a^{\prime}, h(p, a)-h\left(p, a^{\prime}\right) \geq h\left(p^{\prime}, a\right)-h\left(p^{\prime}, a^{\prime}\right)$. Why does this imply positive assortative matching, irrespective of the distribution of types? Usually, the argument is made by noticing that the total output among the four types is maximized (a necessary condition of equilibrium in the TU case, but not, we should emphasize, in the case of NTU) when $p$ matches with $a$ and $p^{\prime}$ with $a^{\prime}$ : this is evident from rearranging the ID condition. 
However, it is more instructive to analyze this from the equilibrium point of view. Suppose that $a$ and $a^{\prime}$ compete for the right to match with $p$ rather than $p^{\prime}$. The increasing difference condition says that $a$ can outbid $a^{\prime}$ in this competition, since the incremental output produced if $a$ switches to $p$ exceeds that when $a^{\prime}$ switches. In particular, this is true whatever the level of utility $u$ that $p^{\prime}$ might be receiving: rewrite (strict) ID as $h(p, a)-\left[h\left(p^{\prime}, a\right)-u\right]>h\left(p, a^{\prime}\right)-\left[h\left(p^{\prime}, a^{\prime}\right)-u\right]$; this is literally the statement that $a$ 's willingness to pay for $p$, given that $p^{\prime}$ is getting $u$, exceeds $a^{\prime \prime}$ s. Thus a situation in which $p$ is matched with $a^{\prime}$ and $p^{\prime}$ with $a$ is never stable: $a$ will be happy to offer more to $p$ than the latter is getting with $a^{\prime}$. The ID result is distribution free: the type distribution will affect the equilibrium payoffs, but the argument just given shows that $p$ 's partner must be larger than $p^{\prime}$ 's regardless of what those payoffs might be.

The convenient feature of TU is that if $a$ outbids $a^{\prime}$ at one level of $u$, he does so for all $u$. Such is not the case with NTU. Our sufficient condition will require explicitly that $a$ can outbid $a^{\prime}$ for all relevant levels of $u$. If this requirement seems strong, recall that the nature of the result sought, namely monotone matching regardless of the distribution, is also strong. By the same token, it is weaker than ID, and includes TU as a special case.

In an NTU model, the division of the surplus between the partners cannot be separated from the level that they generate. Switching to a higher type partner may not be attractive if it is also more costly to transfer utility to a high type, that is, if the frontier is steeper. A sufficient condition for PAM is that not only there is the usual type-type complementarity in the production of surplus, but also there is a type-payoff complementarity: frontiers are flatter, as well as higher, for higher types. This will perhaps be more apparent from the local form of our conditions.

\subsection{Generalized Difference Conditions}

Let $p>p^{\prime}$ and $a>a^{\prime}$ and suppose that $p^{\prime}$ were to get $u$. Then the above reasoning would suggest that $a$ would be able to outbid $a^{\prime}$ for $p$ given that $p^{\prime}$ has a given outside 
option of $u$ :

$$
\phi\left(p, a, \psi\left(a, p^{\prime}, u\right)\right) \geq \phi\left(p, a^{\prime}, \psi\left(a^{\prime}, p^{\prime}, u\right)\right) .
$$

The left-hand side is a's willingness to "pay" (in utility terms) for $p$, given that $p^{\prime}$ receives $u: a$ then receives $v=\psi\left(a, p^{\prime}, u\right)$, so $p$ would get $\phi(p, a, v)$ if matched with $a$. The right-hand side is the counterpart expression for $p^{\prime}: a^{\prime}$ receives $v^{\prime}=\psi\left(a^{\prime}, p^{\prime}, u\right)$, so $p$ would get $\phi\left(p, a^{\prime}, v^{\prime}\right)$ if matched with $a^{\prime} .^{6}$

When satisfied by any $p>p^{\prime}$, and $a>a^{\prime}$, and $u$ feasible for $\left(p^{\prime}, a\right)$, condition (4.1) is called Generalized Increasing Differences (GID). The concept is illustrated in Figure 4.1. The frontiers for the matched pairs $\left\langle p^{\prime}, a^{\prime}\right\rangle,\left\langle p^{\prime}, a\right\rangle,\langle p, a\rangle$, and $\left\langle p, a^{\prime}\right\rangle$ are plotted in a four-axis diagram. The compositions in (4.1) are indicated by following the arrows around from a level of utility $u$ for $p^{\prime}$. Note that the utility $p$ obtains on the " $a$ side" exceeds that on the $a^{\prime}$ side of the diagram. So an equivalent way to state the condition is that whenever a low type $p^{\prime}$ is indifferent between two agents $a$ and $a^{\prime}$, a higher type $p$ will (weakly) prefer the higher type $a$ when he guarantees both agents the same feasible payoffs that they would obtain with $p^{\prime}$ :

$$
\phi\left(p^{\prime}, a^{\prime}, v^{\prime}\right)=\phi\left(p^{\prime}, a, v\right) \Rightarrow \phi(p, a, v) \geq \phi\left(p, a^{\prime}, v^{\prime}\right) .
$$

GID is thus a kind of a single-crossing condition, and as we have mentioned and will show below, is an ordinal property.

Our main result states that GID is sufficient for PAM in the sense that all equilibria are payoff equivalent to a PAM equilibrium. There is an analogous condition, Generalized Decreasing Differences (GDD), for NAM.

Proposition 1. (i) A sufficient condition for an economy to satisfy PAM is that $\phi$

\footnotetext{
${ }^{6}$ Obviously the reasoning can be made in terms of $p$ and $p^{\prime}$ competing for $a$; in this case the (GID) condition is

$$
\psi\left(a, p, \phi\left(p, a^{\prime}, v\right)\right) \geq \psi\left(a, p^{\prime}, \phi\left(p^{\prime}, a^{\prime}, v\right)\right)
$$

it is straightforward to verify that the two conditions are equivalent. In applications, it may be easier to verify the condition from the point of view of one side rather than the other.
} 


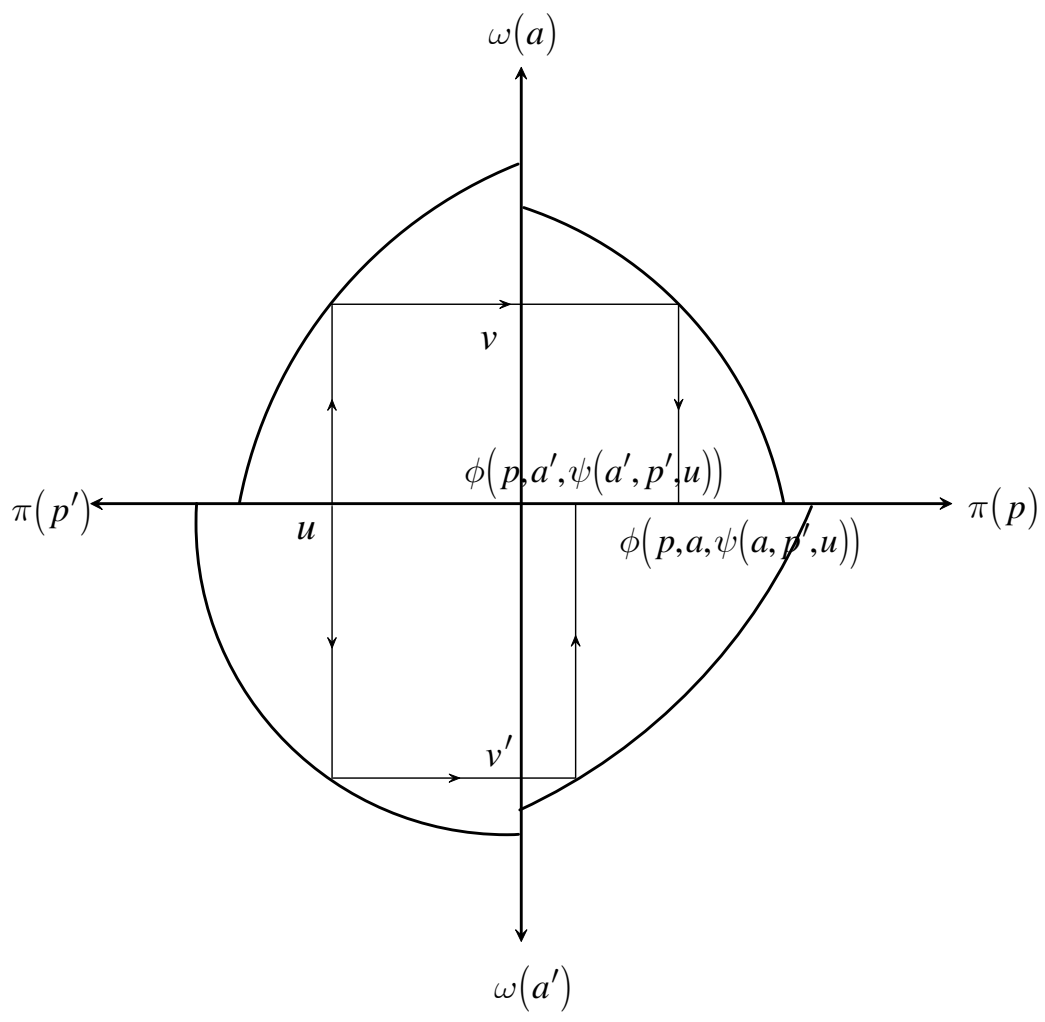

Figure 4.1: Generalized Increasing Differences

satisfies generalized increasing differences (GID) : whenever $p>p^{\prime}, a>a^{\prime}$, and $u \in$ $\left[0, \phi\left(p^{\prime}, a, 0\right)\right], \phi\left(p, a, \psi\left(a, p^{\prime}, u\right)\right) \geq \phi\left(p, a^{\prime}, \psi\left(a^{\prime}, p^{\prime}, u\right)\right)$.

(ii) A sufficient condition for an economy to satisfy NAM is that $\phi$ satisfies generalized decreasing differences (GDD) : whenever $p>p^{\prime}, a>a^{\prime}$, and $u \in\left[0, \phi\left(p^{\prime}, a^{\prime}, 0\right)\right]$, $\phi\left(p, a, \psi\left(a, p^{\prime}, u\right)\right) \leq \phi\left(p, a^{\prime}, \psi\left(a^{\prime}, p^{\prime}, u\right)\right)$.

The basic logic of the proof is very simple, particularly when GID holds strictly. Suppose that contrary to PAM there is an equilibrium with types $p>p^{\prime}$ and $a>a^{\prime}$ that are matched negatively, i.e., $\left\langle p^{\prime}, a\right\rangle$ and $\left\langle p, a^{\prime}\right\rangle$. Feasibility entails that $\pi\left(p^{\prime}\right) \leq \phi\left(p^{\prime}, a, 0\right)$, so by strict GID,

$$
\phi\left(p, a, \psi\left(a, p^{\prime}, \pi\left(p^{\prime}\right)\right)\right)>\phi\left(p, a^{\prime}, \psi\left(a^{\prime}, p^{\prime}, \pi\left(p^{\prime}\right)\right)\right) .
$$

Stability requires that $a^{\prime}$ does not want to switch to $p^{\prime}$, and $a$ does not want to switch 
to $p$ :

$$
\begin{aligned}
& \omega\left(a^{\prime}\right)=\psi\left(a^{\prime}, p, \pi(p)\right) \geq \psi\left(a^{\prime}, p^{\prime}, \pi\left(p^{\prime}\right)\right) \\
& \omega(a)=\psi\left(a, p^{\prime}, \pi\left(p^{\prime}\right)\right) \geq \psi(a, p, \pi(p))
\end{aligned}
$$

Applying $\phi\left(p, a^{\prime}, \cdot\right)$ to both sides of the first inequality and $\phi(p, a, \cdot)$ to the second yields (the inequalities are reversed since the operators are decreasing)

$$
\begin{aligned}
\pi(p) & =\phi\left(p, a^{\prime}, \psi\left(a^{\prime}, p, \pi(p)\right)\right) \leq \phi\left(p, a^{\prime}, \psi\left(a^{\prime}, p^{\prime}, \pi\left(p^{\prime}\right)\right)\right) \\
\phi\left(p, a, \psi\left(a, p^{\prime}, \pi\left(p^{\prime}\right)\right)\right) & \leq \phi(p, a, \psi(a, p, \pi(p))) \leq \pi(p) ;
\end{aligned}
$$

thus $\phi\left(p, a, \psi\left(a, p^{\prime}, \pi\left(p^{\prime}\right)\right)\right) \leq \phi\left(p, a^{\prime}, \psi\left(a^{\prime}, p^{\prime}, \pi\left(p^{\prime}\right)\right)\right)$, contradicting strict GID. The full proof considers the general case in which GID doesn't hold strictly and where there may exist equilibria which do not satisfy PAM; in this case we show that there is a payoff equivalent PAM equilibrium. Details are in the Appendix.

\subsection{The Differentiable Case}

We now present a set of cross-partial derivative conditions. For this subsection, suppose that $P$ and $A$ are nondegenerate closed intervals. Let

$$
D=\left\{(p, a, v) \in \mathbb{R}^{3}: p \in P, a \in A, v \in(0, \psi(a, p, 0))\right\}
$$

be the "domain of nondegeneracy" of $\phi$, that is for each pair $(p, a)$, we ignore the corner values 0 and $\psi(a, p, 0)$.

We shall also assume that each agent benefits from matching with a higher type partner.

Definition 3. $\phi$ is type increasing if for all $(p, a) \in P \times A, u, v \in \mathbb{R}_{+}, \phi(p, a, v)$ is nondecreasing in $a$ and $\psi(a, p, u)$ is nondecreasing in $p$.

Note that $\phi$ type increasing implies that $\phi$ and $\psi$ are nondecreasing in own type as well as partner type. ${ }^{7}$

\footnotetext{
${ }^{7}$ Consider $\hat{a}>a$. By definition, for $u \in[0, \phi(p, a, 0)]$, we have $u=\phi(p, a, \psi(a, p, u))=$
} 
Corollary 1. Suppose $\phi$ is type increasing and twice continuously differentiable on $D$.

(i) A sufficient condition for the economy to satisfy PAM is that for all $(p, a, v) \in D$,

$$
\phi_{12}(p, a, v) \geq 0 \text { and } \phi_{13}(p, a, v) \geq 0 .
$$

(ii) A sufficient condition for the economy to satisfy $N A M$ is that for all $(p, a, v) \in D$,

$$
\phi_{12}(p, a, v) \leq 0 \text { and } \phi_{13}(p, a, v) \leq 0 .
$$

The proof exploits the cross partial assumptions and the fact that $\phi$ is type increasing to ensure that the marginal value of own type $\phi_{1}\left(\hat{p}, a, \psi\left(a, p^{\prime}, u\right)\right)$ is monotone in $a$ for $\hat{p} \in\left[p^{\prime}, p\right]$, and then integrates over $\hat{p}$ to obtain the GDC conditions. See the Appendix.

Obviously, with TU, $\phi_{13}=0$, so (4.3) reduces to the standard condition in that case. The extra term reflects the fact that changing the type results in a change in the slope of the frontier. For PAM, the idea is that higher types can transfer utility to their partners more easily ( $\phi_{3}$ is less negative, hence flatter).

The conditions in Corollary 1 illustrate the separate roles of both the usual type-type complementarity and the type-payoff complementarity we have mentioned. In terms of the bidding story we mentioned in Section 4.1, if two different types are competing for a higher type partner, both will be willing to offer her more than they would a partner with a lower type $\left(\phi_{2}>0\right)$; if the higher type's frontier is flatter than the lower's frontier $\left(\phi_{13} \geq 0\right)$, it will cost the higher type less to do this than it will the lower one; meanwhile if the high type is also more productive on the margin $\left(\phi_{12}>0\right)$ then he is sure to win, in effect being both more productive and having lower costs.

Remark 1. The GID condition states that whenever $p>p^{\prime}$ and $u \in\left[0, \phi\left(p^{\prime}, a, 0\right)\right]$, the function $\phi\left(p, a, \psi\left(a, p^{\prime}, u\right)\right)$ is nondecreasing in $a$. Thus it is (almost) immediate that $\phi(p, \hat{a}, \psi(\hat{a}, p, u))$. Since $\phi$ is nondecreasing in $a, \phi(p, a, \psi(a, p, u)) \leq \phi((p, \hat{a}, \psi(a, p, u))$, hence $\phi(p, \hat{a}, \psi(\hat{a}, p, u)) \leq \phi(p, \hat{a}, \psi(a, p, u))$ which implies that $\psi(\hat{a}, p, u) \geq \psi(a, p, u)$ since $\phi$ is decreasing in payoff. 
if $\phi$ is type increasing and continuously differentiable on $D$, a necessary and sufficient condition for GID is

$$
\phi_{2}\left(p, a, \psi\left(a, p^{\prime}, u\right)\right)+\phi_{3}\left(p, a, \psi\left(a, p^{\prime}, u\right)\right) \cdot \psi_{1}\left(a, p^{\prime}, u\right) \geq 0
$$

for all types $p, p^{\prime} \in P$ with $p \geq p^{\prime}$ and all $a \in A$ and utilities $u \in\left(0, \phi\left(p^{\prime}, a, 0\right)\right.$ ) (see Appendix). However, it appears that in practice, this condition would be more difficult to verify than (4.3).

\section{Applications}

In this section we present two examples that are representative of those considered in the literature and use them to illustrate the application of our general results. The first is a marriage market model in which partners vary in risk attitude and must share risks within their households. The second is a principal-agent model in which agents vary by wealth and principals by project risk.

\subsection{Risk Sharing in Households}

Consider a marriage market model in which the primary desideratum in choosing a mate is suitability for risk sharing. There are two sides to the market for households, and we denote by $p$ the characteristics of the men and by $a$ the characteristics of the women. Household production is random, with two possible outcomes $w_{2}>w_{1}>0$, and associated probabilities $q_{2}$ and $q_{1}$. Everyone is an expected utility maximizer; income $y$ yields utility $U(p, y)$ to a man of type $p$ and $V(a, y)$ to a woman of type $a$. Unmatched agents get utility zero. For all $p$ and $a, U$ and $V$ are twice differentiable, strictly increasing, and strictly concave in income, with the marginal utility of income becoming unbounded as income approaches zero. The characteristics $p$ and $a$ are interpreted as the indices of absolute of risk tolerance: if $p>p^{\prime}$, then $-U_{22}(p, y) / U_{2}(p, y)<-U_{22}\left(p^{\prime}, y\right) / U_{2}\left(p^{\prime}, y\right)$ for all $y$, and $a>a^{\prime}$ implies $-V_{22}(a, y) / V_{2}(a, y)<-V_{22}\left(a^{\prime}, y\right) / V_{2}\left(a^{\prime}, y\right)$ for all $y$. By Pratt's theorem, $U(p, \cdot)$ is a strict convexification of $U\left(p^{\prime}, \cdot\right)$, and $V(a, \cdot)$ is a strict convexification of $V\left(a^{\prime}, \cdot\right)$. 
For informational or enforcement reasons, the only risk sharing possibilities in this economy lie within a household consisting of two agents. When partners match, their (explicit or implicit) contract $\left\{s_{i}\right\}_{i=1,2}$ specifies how each realization of the output will be shared between them: $s_{i}$ goes to the woman in state $i$, and the remaining $w_{i}-s_{i}$ goes to the man.

For a household $(\hat{p}, a)$, the maximum expected utility the man can achieve if the woman requires expected utility $v$ is given by the value $\phi$ of the optimal risk sharing problem:

$$
\phi(\hat{p}, a, v) \equiv \max _{\left\{s_{i}\right\}_{i=1,2}} \Sigma_{i} q_{i} U\left(\hat{p}, w_{i}-s_{i}\right) \text { s.t. } \Sigma_{i} q_{i} V\left(a, s_{i}\right) \geq v .
$$

Call the solution $s^{\hat{p} a}$ for a match $\langle\hat{p}, a\rangle$ when $a$ gets $v$ and $s^{\hat{p} a^{\prime}}$ for a match $\left\langle\hat{p}, a^{\prime}\right\rangle$ when $a^{\prime}$ gets $v^{\prime}$, where $\hat{p} \in\left\{p, p^{\prime}\right\}$. These are illustrated in Figure 5.1. By the first-order conditions and strict concavity of the utilities, we must have

$$
s_{1}<s_{2} \text { and } w_{1}-s_{1}<w_{2}-s_{2}
$$

for any optimal sharing rule $s$. Moreover, it follows from Pratt's theorem, strict concavity, and the first-order conditions that

$$
s^{\hat{p} a} \text { is unique for }\langle\hat{p}, a\rangle ; s^{\hat{p} a} \neq s^{\hat{p} a^{\prime}}, \hat{p} \in\left\{p, p^{\prime}\right\} \text { and } s^{p \hat{a}} \neq s^{p^{\prime} \hat{a}}, \hat{a} \in\left\{a, a^{\prime}\right\} .
$$

We claim that GDD is satisfied (in fact, in its strict form) by this model. We verify that

$$
\phi\left(p^{\prime}, a^{\prime}, v^{\prime}\right)=\phi\left(p^{\prime}, a, v\right) \Rightarrow \phi(p, a, v)<\phi\left(p, a^{\prime}, v^{\prime}\right) .
$$

Assume that $v$ and $v^{\prime}$ are such that $\phi\left(p^{\prime}, a^{\prime}, v^{\prime}\right)=\phi\left(p^{\prime}, a, v\right)$, and suppose first that $\Sigma_{i} q_{i} V\left(a^{\prime}, s^{p a}\right) \geq \Sigma_{i} q_{i} V\left(a^{\prime}, s^{p^{\prime} a^{\prime}}\right)=v^{\prime}$. Then $w-s^{p a}$ is in the feasible set for the problem that determines $w-s^{p a^{\prime}}$, and by revealed preference and (5.3) we have (5.4).

Thus, assume instead that (as in the figure)

$$
\Sigma_{i} q_{i} V\left(a^{\prime}, s^{p a}\right)<\Sigma_{i} q_{i} V\left(a^{\prime}, s^{p^{\prime} a^{\prime}}\right) .
$$

By construction $\Sigma_{i} q_{i} V\left(a, s^{p a}\right)=\Sigma_{i} q_{i} V\left(a, s_{i}^{p^{\prime} a}\right)$; by revealed preference and (5.3), $\Sigma_{i} q_{i} V\left(a, s_{i}^{p^{\prime} a}\right)>\Sigma_{i} q_{i} V\left(a, s_{i}^{p^{\prime} a^{\prime}}\right)$, and therefore,

$$
\Sigma_{i} q_{i} V\left(a, s^{p a}\right)>\Sigma_{i} q_{i} V\left(a, s_{i}^{p^{\prime} a^{\prime}}\right) .
$$




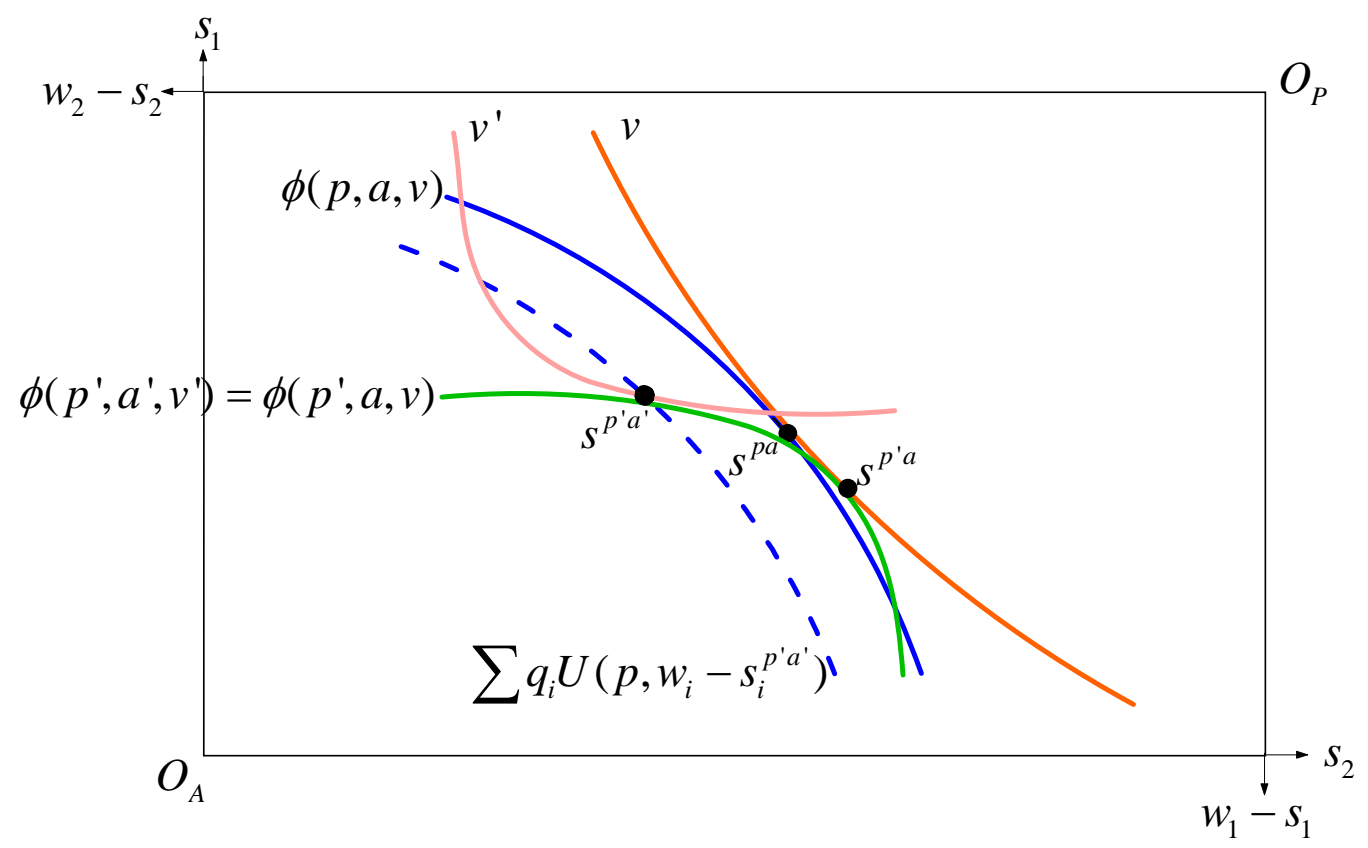

Figure 5.1: Risk Sharing

We show that

$$
s_{1}^{p a}<s_{1}^{p^{\prime} a^{\prime}}<s_{2}^{p^{\prime} a^{\prime}}<s_{2}^{p a}
$$

The middle inequality is from (5.2). Violation of only one of the other inequalities implies that both $a$ and $a^{\prime}$ strictly prefer either $s^{p a}$ or $s^{p^{\prime} a^{\prime}}$ contradicting (5.5) or (5.6). Suppose then that $s_{1}^{p^{\prime} a^{\prime}}<s_{1}^{p a}<s_{2}^{p a}<s_{2}^{p^{\prime} a^{\prime}}$ (the left and right inequalities are strict to avoid contradiction with (5.5) and (5.6)). Since $a^{\prime}$ prefers $s^{p^{\prime} a^{\prime}}$ to $s^{p a}$, the vector of utilities $\left(V\left(a^{\prime}, s_{1}^{p^{\prime} a^{\prime}}\right), V\left(a^{\prime}, s_{2}^{p^{\prime} a^{\prime}}\right)\right)$ is a mean increasing spread of $\left(V\left(a^{\prime}, s_{1}^{p a}\right), V\left(a^{\prime}, s_{2}^{p a}\right)\right)$. As $V(a, \cdot)$ is a strict convexification of $V\left(a^{\prime}, \cdot\right), a$ strictly prefers $s^{p^{\prime} a^{\prime}}$ to $s^{p a}$, contradicting (5.6). This establishes (5.7).

Combining (5.2) and (5.7) gives $w_{1}-s_{1}^{p^{\prime} a^{\prime}}<w_{1}-s_{1}^{p a}<w_{2}-s_{2}^{p a}<w_{2}-s_{2}^{p^{\prime} a^{\prime}}$. By construction $\Sigma_{i} q_{i} U\left(p^{\prime}, w_{i}-s_{i}^{p^{\prime} a^{\prime}}\right)=\Sigma_{i} q_{i} U\left(p^{\prime}, w_{i}-s_{i}^{p^{\prime} a}\right)$; revealed preference and (5.3) imply that $\Sigma_{i} q_{i} U\left(p^{\prime}, w_{i}-s_{i}^{p^{\prime} a}\right)>\Sigma_{i} q_{i} U\left(p^{\prime}, w_{i}-s_{i}^{p a}\right)$. Therefore, $p^{\prime}$ prefers $w-s^{p^{\prime} a^{\prime}}$ to $w-s^{p a}$; and $\left(U\left(p^{\prime}, s_{1}^{p^{\prime} a^{\prime}}\right), U\left(p^{\prime}, s_{2}^{p^{\prime} a^{\prime}}\right)\right)$ is a mean increasing spread of $\left(U\left(p^{\prime}, s_{1}^{p a}\right), U\left(p^{\prime}, s_{2}^{p a}\right)\right)$. Since 
$U(p, \cdot)$ is a strict convexification of $U\left(p^{\prime}, \cdot\right), p$ strictly strictly prefers $w-s^{p^{\prime} a^{\prime}}$ to $w-s^{p a}$, or $\Sigma_{i} q_{i} U\left(p, w_{i}-s_{i}^{p^{\prime} a^{\prime}}\right)>\Sigma_{i} q_{i} U\left(p, w_{i}-s_{i}^{p a}\right)=\phi(p, a, v)$. Finally, by revealed preference, $\phi\left(p, a^{\prime}, v^{\prime}\right) \geq \Sigma_{i} q_{i} U\left(p, w_{i}-s_{i}^{p^{\prime} a^{\prime}}\right)$ and therefore $\phi\left(p, a^{\prime}, v^{\prime}\right)>\phi(p, a, v)$ proving (5.4).

Thus strict GDD is satisfied, and we conclude that in the risk-sharing economy men and women will always match negatively in risk attitude. This is intuitive: a risk-neutral agent is willing to offer a better deal for insurance than is a risk averse one, so those demanding the most insurance (the most risk averse) will share risk with the least risk averse, while the moderately risk averse share with each other. ${ }^{8}$

In case the frontier $\phi$ admits a closed form solution, verification of the GDC is straightforward. For instance, putting $U(p, y)=\log (1+p+y), V(a, y)=\log (1+a+y)$ (type represents initial wealth, which may be shared within the partnership; the number of income realizations can be arbitrary), one obtains $\phi(p, a, v)=\log \left(1-e^{v-\Sigma_{p a}}\right)+\Sigma_{p a}$, where $\Sigma_{p a}$ denotes $\Sigma_{i} q_{i} \log \left(w_{i}+p+a+2\right)$. Then

$$
\phi\left(p, a, \psi\left(a, p^{\prime}, v\right)=\log \left(1-e^{\Sigma_{p^{\prime} a}-\Sigma_{p a}}+e^{v-\Sigma_{p a}}\right)+\Sigma_{p a}\right.
$$

and

$$
\phi\left(p, a^{\prime}, \psi\left(a^{\prime}, p^{\prime}, v\right)=\log \left(1-e^{\Sigma_{p^{\prime} a^{\prime}}-\Sigma_{p a^{\prime}}}+e^{v-\Sigma_{p a^{\prime}}}\right)+\Sigma_{p a^{\prime}} .\right.
$$

Now,

$$
\phi\left(p, a, \phi\left(a, p^{\prime}, v\right)\right)<\phi\left(p, a^{\prime}, \phi\left(a^{\prime}, p^{\prime}, v\right)\right)
$$

if and only if

$$
\left(1-e^{\Sigma_{p^{\prime} a}-\Sigma_{p a}}+e^{v-\Sigma_{p a}}\right) e^{\Sigma_{p a}}<\left(1-e^{\Sigma_{p^{\prime} a^{\prime}}-\Sigma_{p a^{\prime}}}+e^{v-\Sigma_{p a^{\prime}}}\right) e^{\Sigma_{p a^{\prime}}},
$$

that is if $e^{\Sigma_{p a}}-e^{\Sigma_{p^{\prime} a}}<e^{\Sigma_{p a^{\prime}}}-e^{\Sigma_{p^{\prime} a^{\prime}}}$. This is just the requirement that the function $e^{\Sigma_{p a}}$ satisfies strict decreasing differences, which it clearly does, since $\frac{\partial^{2}}{\partial p \partial a} e^{\Sigma_{p a}}=$ $-e^{\Sigma_{p a}} \operatorname{Var}\left(\frac{1}{w+p+a+2}\right)<0$.

For this logarithmic case, the local condition (4.4) does not apply: it is easy to check that $\phi_{1}, \phi_{2}>0$ and $\phi_{12}<0$, while $\phi_{13}>0$. However, the model in the next subsection does admit application of the local conditions.

${ }^{8}$ Recently, Chiappori and Reny (2006) have extended this argument to show that strict GDD holds in the case of an arbitrary number of states. 


\subsection{Matching Principals and Agents}

Principals' projects have a common expected return but differ in their risk characteristics; they must match with agents, who differ in initial wealth. Agents have declining absolute risk aversion, and the question is whether the safest projects are tended by the most or the least risk averse, i.e., the poorest or wealthiest agents.

Risk-neutral principals have type indexed by $p \in[\underline{p}, 1]$, and agents have type index $a \in[\underline{a}, \bar{a}]$, where $\underline{p}, \underline{a}>0$. Agents' unobservable effort $e$ can either be 1 or 0 . The principal's type indexes the success yield and probability of his project: it yields $R / p$ with probability $p$ and 0 with probability $1-p$ provided his agent exerts $e=1$; it yields 0 with probability 1 if $e=0$. Thus, conditional on high effort, all tasks have the same expected return $R$, but higher $p$ implies lower risk. An agent of type $a$ has utility $V(a+y)$ from income $y$; her type represents initial wealth. $V(\cdot)$ is twice differentiable and unbounded below, $V^{\prime}>0>V^{\prime \prime}$, and it displays increasing absolute risk tolerance.

The frontier for a principal of type $p$ who is matched to an agent of type $a$ is given by

$$
\begin{gathered}
\phi(p, a, v)=\max _{s_{0}, s_{1}} R-p s_{1}-(1-p) s_{0} \\
\text { s.t. } p V\left(a+s_{1}\right)+(1-p) V\left(a+s_{0}\right)-1 \geq V\left(a+s_{0}\right), \\
p V\left(a+s_{1}\right)+(1-p) V\left(a+s_{0}\right)-1 \geq v
\end{gathered}
$$

where $s_{1}$ and $s_{0}$ are the wages paid in case of success and failure respectively. ${ }^{9}$ The first inequality is the incentive compatibility condition that ensures the agent takes high effort.

Intuition might suggest that since wealthier agents are less risk averse, they should be matched to riskier tasks while the more risk averse agents should accept the safer tasks (i.e., there should be NAM in $(p, a))$.

\footnotetext{
${ }^{9}$ In this example, the agents' autarchy payoffs are not zero, at least if it assumed that they can consume their initial wealth. As we note in Section 7.1, this generalization presents no particular difficulty.
} 
But this intuition is incomplete, and indeed misleading, as the following application of Corollary 1 shows. By standard arguments, both constraints bind. Let $C(\cdot) \equiv V^{-1}(\cdot)$. Thus $V\left(a+s_{1}\right)=\frac{1}{p}+V\left(a+s_{0}\right)$, and $V\left(a+s_{0}\right)=v$, from which

$$
\phi(p, a, v)=R+a-p C\left(\frac{1}{p}+v\right)-(1-p) C(v) .
$$

Thus,

$$
\begin{aligned}
\phi_{1}(p, a, v) & =\frac{1}{p} C^{\prime}\left(\frac{1}{p}+v\right)-C\left(\frac{1}{p}+v\right)+C(v) \\
\phi_{2}(p, a, v) & =1 \\
\phi_{12} & =0 \\
\phi_{13}(p, a, v) & =\frac{1}{p} C^{\prime \prime}\left(\frac{1}{p}+v\right)-C^{\prime}\left(\frac{1}{p}+v\right)+C^{\prime}(v) .
\end{aligned}
$$

$\phi_{1}$ is positive since $C^{\prime \prime}>0$, so $\phi$ is type increasing. Notice that if $C^{\prime}$ is convex (equivalently $V^{\prime \prime} / V^{\prime 3}$ is decreasing; see Jewitt, 1988 for a discussion - all utilities of the CRRA class that are more risk averse than square root utility have this property), then $\phi_{13} \geq 0$ : there is PAM in $(p, a)$ : as long as risk aversion does not decline "too quickly," agents with lower risk aversion (higher wealth) are matched to principals with projects that are safer, i.e., more likely to succeed. This result may appear surprising, since empirically we tend to associate (financially) riskier tasks to wealthier workers. ${ }^{10}$

The explanation is that in the standard version of the principal-agent model with utility additively separable in income and effort, incentive compatibility for a given effort level entails that the amount of risk borne by the agent increases with wealth $\left(\frac{d s_{1}}{d s_{0}}>0\right.$ along the incentive compatibility constraint). This effect arises from the diminishing marginal utility of income. Though wealthier agents tolerate risk better than the poor, they must accept more risk on a given task; with $C^{\prime}$ convex, the latter effect dominates, and the wealthy therefore prefer the safer tasks. Put another way, a less risky task allows for a reduction in risk borne by the agent; given the increasing risk effect of incentive compatibility, the benefit of the risk reduction is greater for the rich than for the poor, and this generates a complementarity between safety and wealth.

\footnotetext{
${ }^{10}$ Of course, if risk aversion declines fast enough $-C^{\prime}$ is concave - then the "intuitive" negative matching pattern obtains, though this entails a possibly implausibly high level of risk tolerance.
} 
The result offers a possible explanation for the finding in Ackerberg and Botticini (2002) that in medieval Tuscany, wealthy peasants were more likely than poor peasants to tend safe crops (cereals) rather than risky ones (vines).

This example is instructive because the entire effect comes from the nontransferability of the problem. There is no direct "productive" interaction between principal type and agent type $\left(\phi_{12}=0\right)$; only the type-payoff complementarity plays a role in determining the match.

\section{Ordinality, Necessity, Modularity}

In this section we investigate the strength of the GDC. First, we note that GID and GDD are preserved under ordinal transformations of types' preferences. This implies that the analyst is free to choose whichever representation of preferences is most convenient, and leads to a weakening of the differential conditions. In a number of cases, the NTU model even admits a TU representation, in which case GID and GDD reduce to ID and DD of the joint payoff function induced by the representation. After a brief discussion of necessity, we turn to a comparison of the GDC with well-known lattice theoretic concepts.

\subsection{Ordinality}

The core of an economy, and thus any core matching pattern, is independent of the cardinal representation of preferences. However, some representations may be easier to work with after monotone transformations of types' utilities; in some instances it may be possible to recover a transferable utility representation.

Let $F(u ; p)$ be a strictly increasing transformation applied to type $p$ 's utility $u$ and let $F^{-1}(u ; p)$ be its inverse. Similarly, transform type $a$ 's utility by $G(v ; a)$. Let

$$
\phi^{F, G}(p, a, v)=F\left(\phi\left(p, a, G^{-1}(v ; a)\right) ; p\right)
$$

be the new frontier functions after transformations $F$ and $G$ are applied. We call $\phi^{F, G}$ a representation of $\phi$. Its quasi-inverse is $\psi^{G, F}(a, p, u)=G\left(\psi\left(a, p, F^{-1}(u ; p)\right) ; a\right)$. The 
following result, proved in the Appendix, shows the invariance of the GID condition to ordinal transformations of agents' utilities.

Proposition 2. Suppose GID holds for $\phi$. Then GID holds for any other frontier function generated from $\phi$ by increasing transformations of types' utilities. The same result is true for GDD.

A suitably chosen representation of $\phi$ may be easier to work with than $\phi$ itself. In particular, though the generalized difference conditions are preserved for all representations of $\phi$, not so the differential conditions in Corollary 1 . Hence, while the differential conditions may not hold for $\phi$, they might hold for an alternate representation $\phi^{F, G}$. It is enough that one representation of $\phi$ satisfy condition (4.3) or (4.4) to guarantee monotone matching.

For instance, in the logarithmic version of risk sharing in Section 5.1, transform the utility by exponentiation for all types, i.e., set $F(v ; p)=e^{v}$ and $G(u ; a)=e^{u}$; then $\phi^{F, G}(p, a, v)=e^{\Sigma_{p a}}-v$. Unlike $\phi$, which does not satisfy the conditions in Corollary 4.3, $\phi^{F, G}$ does, since $\phi_{12}^{F, G}<0=\phi_{13}^{F, G}$. Notice that the transformation of payoffs actually yields an expression of the frontiers in a transferable utility form.

Starting with a model $\phi(p, a, v)$, say that it is TU-representable if there is a representation $\phi^{F, G}$ of $\phi$ and a function $h(p, a)$ such that

$$
\forall p, a, v, F(\phi(p, a, v) ; p)=h(p, a)-G(v ; a)
$$

Then $F(\phi(p, a, v) ; p)$ is a TU model, since the transformed payoffs to $p$ and to $a$ sum to $h(p, a)$, independently of the distribution of transformed utility between $p$ and $a$. A consequence of Proposition 2 is that $\phi$ satisfies GID if and only if $h$ satisfies increasing differences.

Corollary 2. Suppose that $\phi$ has a TU representation $\phi^{F, G}$. Then $\phi$ satisfies GID $(G D D)$ if and only if $h$ satisfies ID (DD), where $h$ is defined in (6.1).

A well-known-example of a model that admits a TU representation is the "linearnormal-exponential" version of the Principal-Agent model (Holmström and Milgrom, 
1987) in which a TU representation is found by looking at players' certainty equivalent incomes rather than their expected utilities. ${ }^{11}$

\subsection{Necessity}

It should come as little surprise - and for completeness is established in the Appendix

- that given a frontier function, if every distribution of types gives rise to an economy that satisfies PAM, that frontier function must satisfy GID.

Proposition 3. If the equilibrium outcome is payoff equivalent to PAM (NAM) for every distribution of types, then the frontier function $\phi$ satisfies GID (GDD).

In other words, if GID is not satisfied, there are type distributions for which the matching pattern will not be payoff equivalent to PAM. ${ }^{12}$ If GDD isn't satisfied either, then matching need not be monotone. In some cases, matching will be positive assortative for some type distributions, negative assortative for others, and nonmonotone for others still. See Legros and Newman (2002a) for examples.

\subsection{Comparison with Lattice Theoretic Conditions}

In TU models, supermodularity of $\phi(p, a, v)$ is equivalent to increasing differences of the production function $h(p, a)$, hence to GID. ${ }^{13}$ It is natural to ask whether there is a relationship of GID to supermodularity for NTU models.

\footnotetext{
${ }^{11}$ See for instance Wright (2004) and Serfes (2005) for recent applications to matching. Our principalagent example also has a TU representation if agents' income utility is taken to be logarithmic.

${ }^{12}$ Notice this necessity result does not say that the GID condition must hold if a particular economy has an equilibrium that is (payoff equivalent to) a positively assortative one: the GID inequality need only hold for the types in the support of that economy's distribution and for the equilibrium payoff levels. Of course, in case $\phi$ has a TU representation, then GID is also necessary for PAM in this stronger sense. It is an open question whether the family of frontiers such that GID is necessary for PAM is broader than the class of TU representable models.

${ }^{13}$ A function $f(x)$ defined on a lattice $L$ is supermodular when $x, y \in L$ implies $f(x \wedge y)+f(x \vee y) \geq$ $f(x)+f(y)$. Here, $L \subset \mathbb{R}^{n}$, and $x \wedge y(x \vee y)$ denote the componentwise minimum (maximum) of $x$ and $y$.
} 
It is evident from Corollary 1 that a sufficient condition for GID is that $\phi$ is type increasing and supermodular in $(p, a)$ and in $(p, v)$. The principal interest of this observation is that it enables us to offer sufficient conditions for monotone matching expressed in terms of the fundamentals of the model, rather than in terms of the frontiers. Recalling the notation in footnote 5 , let $U(x, p, a)$ be the utility function of choice variables $x$ for type- $p$ principal matched to a type- $a$ agent, $V(x, a, p)$ be the corresponding agent's utility, $\Omega(a, p)$ their choice set, and $\Lambda(a, p, v)=\{x: V(x, a, p) \geq v\}$.

A simple application of Theorem 2.7.6 of Topkis (1998) tells us that a sufficient condition for $\phi$ to be type increasing and super- (sub-) modular in $(p, a)$ and $(p, v)$ and therefore for GID (GDD) is that $U$ is super- (sub-) modular and nondecreasing in $(p, a)$, $V$ is nondecreasing in $(a, p)$ and that the set

$$
S_{v}=\{(p, a, x): x \in \Omega(a, b) \cap \Lambda(a, p, v)\}
$$

is a sublattice for each $v$ and the set

$$
S_{a}=\{(p, a, x): x \in \Omega(a, b) \cap \Lambda(a, p, v)\}
$$

a sublattice for each $a$.

Practically speaking, verification/satisfaction of the sublattice property may be difficult. In the case of logarithmic risk sharing of section 5.1, $S_{a}$ is not a sublattice since $U$ is submodular while $\phi$ is supermodular in $(p, v)$. The monotonicity requirements are also strong: in the principal agent example of section 5.2, the objective is not increasing in $p$, though the frontier is. In any case, supermodularity is clearly stronger than GID.

Are there weaker known conditions on frontier that suffice for assortative matching? One such a concept is quasi-supermodularity (QSM), which requires of a function $f$ defined on a lattice $L$ that for any $x, y \in L$,

$$
f(x) \geq f(x \wedge y) \Rightarrow f(x \vee y) \geq f(y)
$$

with strict inequality on the left-hand side implying strict inequality on the right-hand side (Milgrom and Shannon, 1994). Like GID, QSM is a kind of single-crossing condition on products of lattices. 
It is easily verified that quasi-supermodularity (QSM) in $(p, a)$ and in $(p, v)$ is satisfied for all frontiers $\phi$ that satisfy (strict) type-monotonicity. Since strict typemonotonicity is not sufficient for GID (in particular, for TU models, monotonicity of the output function is well-known to be insufficient for PAM; the example in section 2 is a case in point), quasi-supermodularity in $(p, a)$ and in $(p, v)$ is not sufficient for GID, unlike supermodularity in $(p, a)$ and in $(p, v)$.

Nevertheless, when we assume that $\phi(p, a, v)$ is nondecreasing in $a$, quasi-supermodularity of $\phi$ in $(p, a, v)$ implies GID. The two concepts do not coincide, however, as we show in two examples in the Appendix: Example 1 shows that there exist frontiers that are type increasing for which GID holds but not QSM; Example 2 shows that when $\phi$ is decreasing in $a$, there are frontiers satisfying QSM but neither GID nor GDD.

Proposition 4. Consider the set of maps $\phi: P \times A \times \mathbb{R}_{+} \rightarrow \mathbb{R}_{+}$, strictly decreasing in the third argument. If $\phi$ is nondecreasing in its second argument, then QSM of $\phi$ implies GID.

\section{Extensions}

\subsection{Type-Dependent Autarchy Payoffs and Uneven Sides}

Suppose that autarchy generates a payoff $\underline{u}(p)$ to type $p$ and $\underline{v}(a)$ to type $a$; if $\underline{u}(\cdot)$ and $\underline{v}(\cdot)$ are continuous, we can assume without loss of generality that $\underline{u}(\cdot) \geq 0, \underline{v}(\cdot) \geq 0$. Then all the propositions go through as before, since if the generalized difference or differential conditions hold for nonnegative payoffs, they hold on the restricted domain of individually rational ones. Equilibrium will now typically entail that some types remain unmatched, but among those matched, the pattern will be monotone if the appropriate difference condition holds.

By the same token, little changes if the cardinality of the two sides differs. Some agents on the long side will be left out of the match, but the GID condition implies that those who are matched will be positively assorted: our main result implies that those who are matched initially can be rearranged if necessary in a positive assortative fashion 
while receiving the same payoffs. The unmatched agents, who before were unable to underbid any matched agent, will still be unable to do so.

\subsection{One-Sided Models}

One sided models, in which the type space is just $A$, introduce two complications. First they admit a richer variety of monotone matching patterns than two-sided models. ${ }^{14}$. Second, as is well known, they may have existence problems, at least in finite economies. Nevertheless, the apparatus developed here can be adapted straightforwardly to the onesided case to characterize equilibria when they exist.

Now GID takes the form $\phi\left(a^{\prime}, a, \phi\left(a, a^{\prime \prime}, v\right)\right)$ increasing in $a$ for all $v \leq \phi\left(a^{\prime \prime}, a, 0\right), a^{\prime}>$ $a^{\prime \prime}$ (note that $\left.\psi\left(a, a^{\prime}, v\right)=\phi\left(a, a^{\prime}, v\right)\right)$. If GID holds, we obtain a special form of PAM: segregation, wherein matched pairs consist of identical agents (obviously, this requires an even number of agents of each type). The proof mimics that of Proposition 1 in showing that any heterogenous match is unstable if GID holds. Sufficiency of GDD for NAM is shown similarly; we refer the reader to our working paper (Legros and Newman 2002b).

\subsection{Strict NTU}

Our model excludes the extreme case in which the Pareto frontier for a matched pair is a point, as in the literature surveyed in Roth and Sotomayor (1990). Becker (1973) shows that strict monotonicity of the payoffs in type is sufficient for assortative matching in this case. It turns out that while the strict NTU model can be obtained as a limit case of ours, there are discontinuities in the limit. For instance, GID can be satisfied by an economy with strict NTU but not by nearby economies with strictly decreasing frontiers. GID still implies the existence of a PAM equilibrium, but there may also be other equilibria that are not payoff equivalent to a PAM equilibrium. However, if agents are never indifferent between partners, GID is sufficient for an economy to satisfy

\footnotetext{
${ }^{14}$ In the one-sided model, matching satisfies PAM (NAM) if for any two matched pairs $\left\langle a, a^{\prime}\right\rangle$ and $\left\langle\hat{a}, \hat{a}^{\prime}\right\rangle, \max \left\{a, a^{\prime}\right\}>\max \left\{\hat{a}, \hat{a}^{\prime}\right\} \Longrightarrow \min \left\{a, a^{\prime}\right\} \geq(\leq) \min \left\{\hat{a}, \hat{a}^{\prime}\right\}$.
} 
PAM, which weakens Becker's condition. See Legros and Newman (2006a), which also shows that GID is a strengthening of Clark's (2006) and Eeckout's (2000) conditions for uniqueness of equilibrium.

\subsection{Continuum Economies}

Since the GDC conditions are distribution free, our restriction to a finite number of agents is without much loss of generality. When the GID condition is strict, then even in economies with a continuum of agents and/or types, there can never be a negative stable match and PAM is the only equilibrium outcome. When the GID condition holds weakly, then whenever an equilibrium exists, it is possible to construct an equilibrium that is positive assortative. However, to establish the stronger analog of Proposition 1, that is, that any equilibrium is payoff equivalent to an equilibrium satisfying PAM, the algorithm used in the proof of Proposition 1 for finite economies is not applicable and has to be replaced by a limit argument. See Legros and Newman (2006b).

\section{Conclusion}

Many economic situations involving nontransferable utility are naturally modeled as matching or assignment games. We have presented some general sufficient conditions for monotone matching in these models. These have an intuitive basis and appear to be reasonably straightforward to apply. Specifically, if one wants to ensure PAM, it does not suffice only to have complementarity in types; one must ensure as well that there is enough type-payoff complementarity.

Implicitly motivating this paper's focus on properties of the economic environment that lead to monotone matching is the question of how changes in that environment affect matching patterns. Space, not to mention the present state of knowledge, is too limited to offer a complete answer to this question here, but being able to go beyond the TU case is a necessary part of the puzzle. Many phenomena that could be characterized as mass re-assignments of partners can be understood as manifestations of changes to the degree of transferability. 
For instance, mergers and divestitures involve reassignments of say, upstream and downstream divisions of firms. Transferability between divisions depends in part on the efficiency of financial markets, with the magnitude of the effect dependent on characteristics of individual firms such as liquidity position or productivity. Deregulations or innovations in financial markets will typically alter transferability and may lead to widespread reassignment of partnerships between upstream and downstream divisions, i.e., "waves" of corporate reorganization (Holmström and Kaplan, 2001).

Another example is a policy like Title IX, which requires US schools and universities receiving federal funding to spend equally on men's and women's activities (athletic programs having garnered the most public attention), or suffer penalties in the form of lost funding. If one models a college as partnership between a male and female student-athlete, identifying their types with the revenue-generating capacities of their respective sports, the policy acts to transform a TU model into an NTU one, rather like the example of section 2. Imposing Title IX would lead to a reshuffling of the types of males and females who match; the male wrestler (low revenue), formerly matched to the female point guard (high revenue), will now match with, say, a female rower, while the point guard now plays at a football school. There is evidence that this sort of re-assignment has taken place: the oft-noted terminations and contractions of some sports at some colleges are ameliorated by start-ups and expansions at others.

\section{Appendix}

\subsection{Proof of Proposition 1}

We prove (1), the proof of (2) is similar. The proof has two steps. We first establish that when GID holds, any equilibrium matching four types in a NAM fashion has the property that we can rematch these types in a PAM fashion without violating feasibility. We then show that if an equilibrium does not satisfy PAM we can use recursively the previous result to construct a type payoff equivalent equilibrium that satisfies PAM.

Lemma 1. Suppose that GID holds. Consider an equilibrium and associated stable 
$\langle\mathfrak{M}, \pi, \omega\rangle$. If $a^{\prime} \in \mathfrak{M}(p), a \in \mathfrak{M}\left(p^{\prime}\right)$, where $a>a^{\prime}$ and $p>p^{\prime}$, then $\pi(p)=\phi(p, a, \omega(a))$, $\omega(a) \leq \psi(a, p, 0)$ and $\pi\left(p^{\prime}\right)=\phi\left(p^{\prime}, a^{\prime}, \omega\left(a^{\prime}\right)\right)$ and $\omega\left(a^{\prime}\right) \leq \psi\left(a^{\prime}, p^{\prime}, 0\right)$.

Proof. Consider a NAM matching as in the Lemma with payoffs $\pi$ and $\omega$. Feasibility entails

$$
\begin{aligned}
\pi(p) & =\phi\left(p, a^{\prime}, \omega\left(a^{\prime}\right)\right) \text { and } \omega\left(a^{\prime}\right) \leq \psi\left(a^{\prime}, p, 0\right) \\
\pi\left(p^{\prime}\right) & =\phi\left(p^{\prime}, a, \omega(a)\right) \text { and } \omega(a) \leq \psi\left(a, p^{\prime}, 0\right)
\end{aligned}
$$

Stability of the NAM matching requires

$$
\begin{aligned}
\omega\left(a^{\prime}\right) & \geq \psi\left(a^{\prime}, p^{\prime}, \pi\left(p^{\prime}\right)\right) \\
\pi(p) & \geq \phi(p, a, \omega(a)) .
\end{aligned}
$$

From (9.4) and (9.2),

$$
\pi(p) \geq \phi(p, a, \omega(a))=\phi\left(p, a, \psi\left(a, p^{\prime}, \pi\left(p^{\prime}\right)\right)\right)
$$

From $(9.2), \pi\left(p^{\prime}\right) \leq \phi\left(p^{\prime}, a, 0\right)$ and by GID,

$$
\phi\left(p, a, \psi\left(a, p^{\prime}, \pi\left(p^{\prime}\right)\right)\right) \geq \phi\left(p, a^{\prime}, \psi\left(a^{\prime}, p^{\prime}, \pi\left(p^{\prime}\right)\right)\right) .
$$

From (9.3) and (9.1),

$$
\phi\left(p, a^{\prime}, \psi\left(a^{\prime}, p^{\prime}, \pi\left(p^{\prime}\right)\right)\right) \geq \phi\left(p, a^{\prime}, \omega\left(a^{\prime}\right)\right)=\pi(p)
$$

Hence, from (9.5)-(9.7), we must have an equality everywhere, or

$$
\pi(p)=\phi(p, a, \omega(a))=\phi\left(p, a^{\prime}, \psi\left(a^{\prime}, p^{\prime}, \pi\left(p^{\prime}\right)\right)\right)=\phi\left(p, a^{\prime}, \omega\left(a^{\prime}\right)\right)=\pi(p) .
$$

Case 1: $\pi(p)>0$. In (9.8), $\pi(p)=\phi(p, a, \omega(a))$ shows that $(\pi(p), \omega(a))$ is feasible for $(p, a)$; since $\phi\left(p, a^{\prime}, \psi\left(a^{\prime}, p^{\prime}, \pi\left(p^{\prime}\right)\right)\right)>0$, it follows that $\psi\left(a^{\prime}, p^{\prime}, \pi\left(p^{\prime}\right)\right)=\omega\left(a^{\prime}\right) \leq$ $\psi\left(p, a^{\prime}, 0\right)$ and therefore $\left(\pi\left(p^{\prime}\right), \omega\left(a^{\prime}\right)\right)$ is feasible for $\left(p^{\prime}, a^{\prime}\right)$.

Case 2: $\pi(p)=0$. Then $\omega\left(a^{\prime}\right)=\psi\left(a^{\prime}, p, 0\right)>0$, and by stability, $\omega(a) \geq \psi(a, p, 0)$.

We show that we cannot have $\omega(a)>\psi(a, p, 0)$. If we do, define $u_{0}=\phi\left(p^{\prime}, a, \psi(a, p, 0)\right)$.

Then $\phi\left(p^{\prime}, a, 0\right)>u_{0}>\phi\left(p^{\prime}, a, \omega(a)\right)=\pi\left(p^{\prime}\right)$. By construction, $\left.\phi\left(p, a, \psi\left(a, p^{\prime}, u_{0}\right)\right)\right)=0$. 
If $u_{0} \leq \phi\left(p^{\prime}, a^{\prime}, 0\right)$, then $\psi\left(a^{\prime}, p^{\prime}, u_{0}\right)<\psi\left(a^{\prime}, p^{\prime}, \pi\left(p^{\prime}\right)\right) \leq \omega\left(a^{\prime}\right)$ by $(9.3)$; if $u_{0}>\phi\left(p^{\prime}, a^{\prime}, 0\right)$ then $\psi\left(a^{\prime}, p^{\prime}, u_{0}\right)=0<\omega\left(a^{\prime}\right)$. Thus $\phi\left(p, a^{\prime}, \psi\left(a^{\prime}, p^{\prime}, u_{0}\right)\right)>\phi\left(p, a^{\prime}, \omega\left(a^{\prime}\right)\right)=\pi(p)=0=$ $\left.\phi\left(p, a, \psi\left(a, p^{\prime}, u_{0}\right)\right)\right)$, and we have a contradiction of GID.

Therefore $\omega(a)=\psi(a, p, 0)$ and $(0, \omega(a))$ is feasible for $(p, a)$. From $(9.8), \phi\left(p, a^{\prime}, \psi\left(a^{\prime}, p^{\prime}, \pi\left(p^{\prime}\right)\right)\right)=$ 0 , so $\psi\left(a^{\prime}, p^{\prime}, \pi\left(p^{\prime}\right)\right) \geq \psi\left(a^{\prime}, p, 0\right)=\omega\left(a^{\prime}\right)$. But by stability (9.3) we must have an equality: $\psi\left(a^{\prime}, p^{\prime}, \pi\left(p^{\prime}\right)\right)=\omega\left(a^{\prime}\right)$, i.e., $\left(\pi\left(p^{\prime}\right), \omega\left(a^{\prime}\right)\right)$ is feasible for $\left(p^{\prime}, a^{\prime}\right)$.

We have shown that under GID, if in equilibrium there are two matches that violate PAM, it is possible to reassign partners in order to obtain PAM without modifying payoffs and therefore without violating stability. This falls short, however, of showing that starting from an equilibrium in which $\mathfrak{M}$ does not satisfy PAM we can find a payoff equivalent equilibrium satisfying PAM.

The next part of the proof shows an algorithm to construct a new equilibrium $\left\langle\mathfrak{M}^{\prime}, \pi, \omega\right\rangle$ where $\mathfrak{M}^{\prime}$ satisfies PAM and $\pi$ and $\omega$ are the initial equilibrium type payoffs. It is easier to develop the argument in reference to agents rather than types. Let $N$ be the cardinalities of $I$ and $J$. Then, we can write $I=\left\{i_{k}, k \in\{1,2, \ldots, N\}\right\}$ and $J=$ $\left\{j_{k}, k \in\{1,2, \ldots, N\}\right\}$. Higher indexes correspond to lower values of the characteristic, that is agent $i_{k}$ has type $p$ and agent $i_{l}$ has type $p^{\prime}$ where $p \geq p^{\prime}$ if and only if $k \leq l$.

We show that under GID, any equilibrium $\left\langle m, \pi^{*}, \omega^{*}\right\rangle$ is payoff equivalent to the equilibrium $\left\langle m^{*}, \pi^{*}, \omega^{*}\right\rangle$ with $m^{*}\left(i_{k}\right)=j_{k}$.

Suppose that in $\left\langle m, \pi^{*}, \omega^{*}\right\rangle, m\left(i_{k}\right)=j_{l}, l \neq k$. Let $k_{0}$ be the first time this situation arises. Hence for all $t \leq k_{0}-1, m\left(i_{t}\right)=m^{*}\left(i_{t}\right)=j_{t}$. Let $i_{r}$ be such that $m\left(i_{r}\right)=j_{k_{0}}$; by construction if $i_{k_{0}}$ has type $p$ and $j_{k_{0}}$ has type $a, j_{l}$ has type $a^{\prime} \leq a$ and $i_{r}$ has type $p^{\prime} \leq p$. By the Lemma, we can match $i_{k_{0}}$ with $j_{k_{0}}$ and $i_{r}$ with $j_{l}$, keep the same payoffs for the four agents without violating feasibility or stability. Doing so we have a new matching $m_{[1]}$

$$
m_{[1]}\left(i_{t}\right)=\left\{\begin{array}{cc}
m^{*}\left(i_{t}\right) & \text { if } t \leq k_{0} \\
m\left(i_{t}\right) & \text { if } t \geq k_{0}+1, t \neq r \\
j_{l} & \text { if } t=r .
\end{array}\right.
$$


If $m_{[1]}=m^{*}$, we are done. Otherwise, let $k_{1}$ be the first index such that $m_{[1]}\left(i_{t}\right) \neq j_{t}$; by construction of $m_{[1]}$, it must be the case that $k_{1} \geq k_{0}+1$. Repeat the previous construction to obtain a new matching function $m_{[2]}$ with $m_{[2]}(t)=m^{*}(t)$ for $t \leq k_{1}$. Repeating this construction, we will have a value $n$ such that $m_{[n]}=m^{*}$. Stability of $m^{*}$ with respect to $\left(\pi^{*}, \omega^{*}\right)$ is inherited from the stability of $m$ with respect to $\left(\pi^{*}, m^{*}\right)$. Hence $\left\langle m^{*}, \pi^{*}, \omega^{*}\right\rangle$ is an equilibrium satisfying PAM, concluding the proof.

\subsection{Proof of Corollary 1}

Consider $a>a^{\prime}, p>p^{\prime}$ and $u \leq \phi\left(p^{\prime}, a, 0\right)$. By type monotonicity $\phi\left(p^{\prime}, a, 0\right) \geq$ $\phi\left(p^{\prime}, a^{\prime}, 0\right)$.

If $u \in\left[\phi\left(p^{\prime}, a^{\prime}, 0\right), \phi\left(p^{\prime}, a, 0\right)\right], \phi\left(p, a, \psi\left(a, p^{\prime}, u\right)\right)=\phi(p, a, 0)$ which is greater than $\phi\left(p, a^{\prime}, 0\right)$ by type monotonicity. Hence, $\phi\left(p, a, \psi\left(a, p^{\prime}, u\right)\right) \geq \phi\left(p, a^{\prime}, \psi\left(a^{\prime}, p^{\prime}, u\right)\right)$.

If $u \in\left(0, \phi\left(p^{\prime}, a^{\prime}, 0\right)\right)$, since $\phi$ is type monotonic, when $\hat{a} \geq a^{\prime}$, and $\hat{p} \geq p^{\prime}$, $\phi\left(p^{\prime}, a^{\prime}, 0\right) \leq \phi(\hat{p}, \hat{a}, 0)$, therefore $u<\phi(\hat{p}, \hat{a}, 0)$. Letting $v=\psi\left(a^{\prime}, p^{\prime}, u\right),(\hat{p}, \hat{a}, v) \in D$ and $\phi_{12}(\hat{p}, \hat{a}, v)$ is well defined for all $\hat{a} \geq a^{\prime}$ and $\hat{p} \geq p^{\prime}$. Therefore,

$$
\begin{aligned}
0 & \leq \int_{a^{\prime}}^{a} \phi_{12}(\hat{p}, \hat{a}, v) d \hat{a} \\
& =\phi_{1}(\hat{p}, a, v)-\phi_{1}\left(\hat{p}, a^{\prime}, v\right) .
\end{aligned}
$$

Since $\phi_{13} \geq 0$, we have for all $\hat{p} \geq p^{\prime}, \phi_{1}\left(\hat{p}, a, \psi\left(a, p^{\prime}, u\right)\right) \geq \phi_{1}\left(\hat{p}, a, \psi\left(a^{\prime}, p^{\prime}, u\right)\right)$ which together with (9.9) leads to

$$
\phi_{1}\left(\hat{p}, a, \psi\left(a, p^{\prime}, u\right)\right) \geq \phi_{1}\left(\hat{p}, a^{\prime}, \psi\left(a^{\prime}, p^{\prime}, u\right)\right) .
$$

Integrating both sides with respect to $\hat{p}$ on the interval $\left[p^{\prime}, p\right]$ leads to

$\phi\left(p, a, \psi\left(a, p^{\prime}, u\right)\right)-\phi\left(p^{\prime}, a, \psi\left(a, p^{\prime}, u\right)\right) \geq \phi\left(p, a^{\prime}, \psi\left(a^{\prime}, p^{\prime}, u\right)\right)-\phi\left(p^{\prime}, a^{\prime}, \psi\left(a, p^{\prime}, u\right)\right)$.

Since $\phi\left(p^{\prime}, a, \psi\left(a, p^{\prime}, u\right)\right)$ and $\phi\left(p^{\prime}, a^{\prime}, \psi\left(a, p^{\prime}, u\right)\right)$ are both equal to $u$, we obtain the GID condition.

If $u=0$, consider a sequence $\left\{u_{k}\right\} \subset\left(0, \phi\left(p^{\prime}, a^{\prime}, 0\right)\right)$ converging to 0 . For each $k$, we have by the previous case $\phi\left(p, a, \psi\left(a, p^{\prime}, u_{k}\right)\right) \geq \phi\left(p, a^{\prime}, \psi\left(a^{\prime}, p^{\prime}, u_{k}\right)\right)$; since $\phi$ and $\psi$ are continuous, taking the limit on both sides with respect to $k$ yields $\phi\left(p, a, \psi\left(a, p^{\prime}, 0\right)\right) \geq$ $\phi\left(p, a^{\prime}, \psi\left(a^{\prime}, p^{\prime}, 0\right)\right)$. 


\subsection{Proof of Claim in Remark 1}

Suppose GID and let $u \in\left(0, \phi\left(p^{\prime}, a, 0\right)\right)$. Since $\left(p^{\prime}, a, \psi\left(a, p^{\prime}, u\right)\right) \in D$, type monotonicity implies that for all $p>p^{\prime},\left(p, a, \psi\left(a, p^{\prime}, u\right)\right) \in D$. If GID holds, $\phi\left(p, a, \psi\left(a, p^{\prime}, u\right)\right)$ is nondecreasing in $a$ for $p>p^{\prime}$; since this function is differentiable in $a$, we must have $0 \leq \frac{d}{d a} \phi\left(p, a, \psi\left(a, p^{\prime}, u\right)\right)=\phi_{2}\left(p, a, \psi\left(a, p^{\prime}, u\right)\right)+\phi_{3}\left(p, a, \psi\left(a, p^{\prime}, u\right)\right) \cdot \psi_{1}\left(a, p^{\prime}, u\right)$. This proves necessity.

For sufficiency, consider $a^{\prime}<a, p^{\prime}<p$ and $u \leq \phi\left(p^{\prime}, a, 0\right)$. If $u \in\left[\phi\left(p^{\prime}, a^{\prime}, 0\right), \phi\left(p^{\prime}, a, 0\right)\right]$, GID holds since $\phi\left(p, a, \psi\left(a, p^{\prime}, u\right)\right)=\phi(p, a, 0)$. If $u \in\left(0, \phi\left(p^{\prime}, a^{\prime}, 0\right)\right)$, since $\phi$ is type increasing, for all $\hat{a} \geq a, \psi\left(\hat{a}, p^{\prime}, u\right) \leq \psi(\hat{a}, p, u)$ and therefore, $\left(p, \hat{a}, \psi\left(\hat{a}, p^{\prime}, u\right)\right) \in D$, which shows that $\phi\left(p, \hat{a}, \psi\left(\hat{a}, p^{\prime}, u\right)\right)$ is differentiable with respect to $\hat{a}$. Hence, integrating (4.5) on $\left[a^{\prime}, a\right]$ leads to the GID condition. If $u=0$, use a limit argument as in the previous proof.

\subsection{Proof of Proposition 2}

We consider the case for GID; the proof for GDD is similar. It is enough to show that the map $\phi^{F, G}(p, a, v)$ satisfies GID, that is that $\phi^{F, G}\left(p, a, \psi^{G, F}\left(a, p^{\prime}, u\right)\right)$ is increasing in $a$.

$$
\begin{aligned}
\phi^{F, G}\left(p, a, \psi^{G, F}\left(a, p^{\prime}, u\right)\right) & =F\left(\phi\left(p, a, G^{-1}\left(\psi^{G, F}\left(a, p^{\prime}, u\right) ; a\right) ; p\right)\right. \\
& =F\left(\phi\left(p, a, G^{-1}\left(G\left(\psi\left(a, p^{\prime}, F^{-1}\left(u ; p^{\prime}\right)\right) ; a\right) ; a\right) ; p\right)\right. \\
& =F\left(\phi\left(p, a, \psi\left(a, p^{\prime}, F^{-1}\left(u ; p^{\prime}\right)\right) ; p\right)\right.
\end{aligned}
$$

since $F(\cdot ; p)$ is strictly increasing, $\phi^{F, G}\left(p, a, \psi^{G, F}\left(a, p^{\prime}, u\right)\right)$ is increasing in a only if $\phi\left(p, a, \psi\left(a, p^{\prime}, F^{-1}\left(u ; p^{\prime}\right)\right)\right.$ is increasing in $a$, which is true since $\phi$ satisfies GID.

\subsection{Proof of Proposition 3}

Consider PAM, as the case for the necessity of GDD for NAM is similar. Suppose there are $p, p^{\prime} \in P, p>p^{\prime}$ and $a, a^{\prime} \in A, a>a^{\prime}$, and a payoff level $u \leq \phi\left(p^{\prime}, a, 0\right)$ such that $\phi\left(p, a, \psi\left(a, p^{\prime}, u\right)\right)<\phi\left(p, a^{\prime}, \psi\left(a^{\prime}, p^{\prime}, u\right)\right)$. Then we can find a distribution of types such that there is an equilibrium that is not payoff equivalent to PAM. 
To see this, put an equal number of agents at each of the four types $p, p^{\prime}, a, a^{\prime}$. Then there is $\epsilon>0$ such that $\left\langle p^{\prime}, a\right\rangle$ with payoffs $\left(u, \psi\left(a, p^{\prime}, u\right)\right)$ and $\left\langle p, a^{\prime}\right\rangle$ with payoffs $\left.\left(\phi\left(p, a^{\prime}, \psi\left(a^{\prime}, p^{\prime}, u\right)+\epsilon\right), \psi\left(a^{\prime}, p^{\prime}, u\right)+\epsilon\right)\right)$ is an equilibrium. To verify stability, note that by continuity of $\phi$ in $v$, for $\epsilon$ small enough, $\phi\left(p, a, \psi\left(a, p^{\prime}, u\right)\right)<\phi\left(p, a^{\prime}, \psi\left(a^{\prime}, p^{\prime}, u\right)+\epsilon\right)$. Thus $p$ would be strictly worse off switching to $a$ as long as $a$ receives at least his equilibrium payoff. Similarly $a^{\prime}$ would lose $\epsilon$ by switching to $p^{\prime}$. Finally, the match is not payoff equivalent to PAM because $p$ cannot generate $\phi\left(p, a^{\prime}, \psi\left(a^{\prime}, p^{\prime}, u\right)+\epsilon\right)$ in a match with $a$ without offering $a$ less than $\psi\left(a, p^{\prime}, u\right)$ And no match with PAM could support these payoffs, since the same inequality implies that $p$ cannot generate his equilibrium payoff in a match with $a$.

\subsection{Proof of Proposition 4}

Let $p>p^{\prime}, a>a^{\prime}$. We establish GID, i.e.,

$$
\phi\left(p^{\prime}, a^{\prime}, v^{\prime}\right)=\phi\left(p^{\prime}, a, v\right) \Rightarrow \phi(p, a, v) \geq \phi\left(p, a^{\prime}, v^{\prime}\right)
$$

Suppose $\phi\left(p^{\prime}, a^{\prime}, v^{\prime}\right)=\phi\left(p^{\prime}, a, v\right)$; since $\phi$ is nondecreasing in its second argument and decreasing in its third, we have $v \geq v^{\prime}$. Put $x=\left(p^{\prime}, a, v\right)$ and $y=\left(p, a^{\prime}, v^{\prime}\right)$; then $x \wedge y=\left(p^{\prime}, a^{\prime}, v^{\prime}\right)$ and $x \vee y=(p, a, v)$. We therefore have $\phi(x)=\phi(x \wedge y)$, and by QSM, $\phi(x \vee y) \geq \phi(y)$, i.e., $\phi(p, a, v) \geq \phi\left(p, a^{\prime}, v^{\prime}\right)$, as desired.

\subsection{QSM and the GDC}

\section{Example 1.}

The example shows that within the set of type increasing frontiers, QSM is a stronger concept than GID. The construction resembles that in section 2: starting from a pair of payoffs $f(p \mid a)$ to $p$ and $g(a \mid p)$ to $a$, that serves as a "reference point" for a match $(p, a)$, construct a frontier $\phi(p, a, v)$ by allowing transfers at a rate $\beta$ away from the reference payoff. Consider $p>p^{\prime}, a>a^{\prime}$, with the reference points for the four 
possible matches:

$$
\begin{aligned}
f\left(p \mid a^{\prime}\right) & =g\left(a^{\prime} \mid p\right)=t \\
f\left(p^{\prime} \mid a^{\prime}\right) & =g\left(a^{\prime} \mid p^{\prime}\right)=s \\
f\left(p^{\prime} \mid a\right) & =r, g\left(a \mid p^{\prime}\right)=s \\
f(p \mid a) & =g(a \mid p)=t .
\end{aligned}
$$

where we $s<t<r$. We assume that $\beta=1$ in the match $(p, a)$, hence, $\phi(p, a, v)=$ $\max \{0,2 t-v\}$, and that $\beta$ is "small" for the other matches. In particular, we assume that $t>(1+\beta) s, r \in(t, 2 t-s]$, and $\beta<(t-s) /(t+r)$. Note that $\phi$ is increasing in its first two arguments. See Figure 9.1.

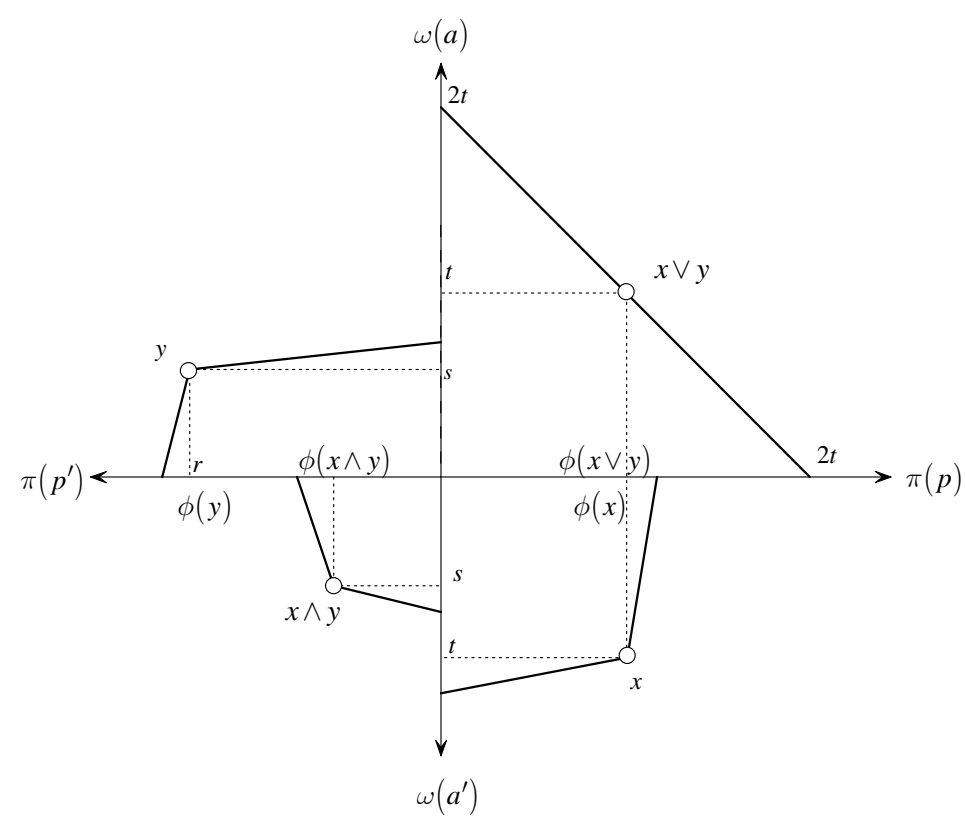

Figure 9.1: GID holds but QSM is violated

We show that QSM is violated. Let $x=\left(p, a^{\prime}, t\right), y=\left(p^{\prime}, a, s\right)$, we have $x \wedge y=$ $\left(p^{\prime}, a^{\prime}, s\right)$ and $x \vee y=(p, a, t)$. Then, $\phi(x)=t>s=\phi(x \wedge y)$ and QSM would require that $\phi(x \vee y)>\phi(y)$. However, $\phi(y)=r$ and $\phi(x \vee y)=t$, and since $r>t$ we have a violation of QSM. 
We now verify GID. For $u \in\left[0, \phi\left(p^{\prime}, a, 0\right)\right]$, where $\phi\left(p^{\prime}, a, 0\right)=r+\beta s$, observe that

$$
\phi\left(p, a, \psi\left(a, p^{\prime}, u\right)\right) \geq \phi\left(p, a, \psi\left(a, p^{\prime}, 0\right)\right)=2 t-(s+\beta r) .
$$

On the other hand, $\phi\left(p, a^{\prime}, \psi\left(a^{\prime}, p^{\prime}, u\right)\right) \leq t(1+\beta)$. Therefore, GID holds if

$$
t(1+\beta) \leq 2 t-(s+\beta r)
$$

which is satisfied since $\beta<(t-s) /(t+r)$. This proves that the set of frontiers that are monotonic in types and that satisfy QSM is a strict subset of those satisfying GID.

\section{Example 2.}

The example illustrates the role of the type-increasing condition in establishing Proposition 4: without it, QSM no longer implies GID.

Let $p>p^{\prime}, a>a^{\prime}$, and, where they are positive, $\phi(p, a, v)=s-v, \phi\left(p^{\prime}, a, v\right)=$ $t-v, \phi\left(p^{\prime}, a^{\prime}, v\right)=r-v$, and $\phi\left(p, a^{\prime}, v\right)=s-(s / r) v$, where $0<s<t<r$.

Clearly, $\phi$ is not type increasing. Moreover, for $u \in[0, r], \phi\left(p, a, \psi\left(a, p^{\prime}, u\right)\right)=\max \{0, s-$ $\max (0, t-u)\}$ and $\phi\left(p, a^{\prime}, \psi\left(a^{\prime}, p^{\prime}, u\right)\right)=u s / r$, so that for $u$ in a neighborhood of $t-s$, $\phi$ fails to satisfy GID, while in a neighborhood of $t, \phi$ doesn't satisfy GDD.

We now show that nevertheless, $\phi$ is QSM. Since $\phi$ is nonincreasing in all three arguments, for any choice of $x$ and $y$, we cannot have $\phi(x)>\phi(x \wedge y)$, so it is enough to verify that $\phi(x)=\phi(x \wedge y) \Longrightarrow \phi(x \vee y) \geq \phi(y)$. Note that the QSM inequality holds if $\phi(y)=0$. In all cases below we set $y=(\tilde{p}, \tilde{a}, w)$.

Case 1. $x=\left(p^{\prime}, a^{\prime}, v\right)$. If $v \leq w, x \wedge y=x$ and $x \vee y=y$, and the QSM inequality is satisfied trivially. If $v>w$, then $\phi(x)=\phi(x \wedge y) \Longleftrightarrow \max \{0, r-v\}=\max \{0, r-w\}$, which can happen only if $w \geq r$. Then, $\phi(y)=0$ and the QSM inequality is satisfied.

Case 2. $x=(p, a, v)$. Then, $\phi(x \wedge y)=\phi(\tilde{p}, \tilde{a}, \min (v, w))$. If $v \leq s, \phi(x)=s-v$ and $\phi(x)=\phi(x \wedge y)$ only if $y=x$, and the QSM inequality holds. If $v>s, \phi(x)=0$ and we need $\phi(x \wedge y)=0$, or $\min (v, w) \geq \psi(\tilde{a}, \tilde{p}, 0)$; in this case, $\phi(y)=0$ and the QSM inequality is satisfied. 
Case 3. $x=\left(p^{\prime}, a, v\right)$. Then $\phi(x \wedge y)=\phi\left(p^{\prime}, \tilde{a}, \min (v, w)\right)$. If $v<t, \phi(x)=t-v$ and $\phi(x)=\phi(x \wedge y)$ only if $\tilde{a}=a$ and $v=\min (v, w)$. Then, $y=x \vee y=(\tilde{p}, a, w)$ and the QSM inequality holds. If $v \geq t, \phi(x)=0$ and we need $\phi\left(p^{\prime}, \tilde{a}, \min (v, w)\right)=0$, or $\min (v, w) \geq \psi\left(\tilde{a}, p^{\prime}, 0\right)$. Since $\psi$ is decreasing in types, $\psi(\tilde{a}, \tilde{p}, 0) \leq \psi\left(\tilde{a}, p^{\prime}, 0\right)$, and therefore $\min (v, w) \geq \psi(\tilde{a}, \tilde{p}, 0)$, proving that $\phi(y)=0$ and the QSM inequality holds.

Case 4, $x=\left(p, a^{\prime}, v\right)$, is similar to Case 3 .

Thus QSM is satisfied while neither of the GDC hold: when $\phi$ is not type increasing, QSM has little relationship to the GDC.

\section{References}

[1] Ackerberg, D. and M. Botticini (2002): Endogenous Matching and the Empirical Determinants of Contract Form, Journal of Political Economy, 110, 564-591.

[2] Becker, G.S. (1973): "A Theory of Marriage: Part I, Journal of Political Economy, 81, 813-846.

[3] Besley, T., and M. Ghatak (2005), Competition and Incentives with Motivated Agents, American Economic Review, 95, 616-636.

[4] Chiappori, P.-A. and P. Reny (2006): Matching to Share Risk, Unpublished Manuscript, http://home.uchicago.edu/〜 preny/papers/matching-05-05-06.pdf.

[5] Clark, S. (2006): The Uniqueness of Stable Matchings, Contributions to Theoretical Economics, 6, http://www.bepress.com/bejte/contributions/vol6/iss1/art8

[6] Eeckhout, J. (2000): On the Uniqueness of Stable Marriage Matchings, Economics Letters, 69: 1-8.

[7] Farrell, J., and S. Scotchmer (1988): Partnerships, Quarterly Journal of Economics, 103, 279-297.

[8] Holmström, B., and S.N. Kaplan (2001): Corporate Governance and Merger Activity in the U.S.: Making Sense of the 1980s and 1990s, Journal of Economic Perspectives, 15, 121-144. 
[9] Holmström, B., and P. Milgrom (1987): Aggregation and Linearity in the Provision of Intertemporal Incentives, Econometrica, 55, 303-328.

[10] Jewitt, I. (1988): Justifying the First-Order Approach to the Principal-Agent Problem, Econometrica, 56, 1177-1190.

[11] Kaneko, M. (1982): The Central Assignment Game and the Assignment Markets, Journal of Mathematical Economics, 10, 205-232.

[12] Lazear, E.P. (2000): Performance Pay and Productivity, American Economic Review, 90, 1346-1361.

[13] Legros, P., and A.F. Newman (2002a): "Monotone Matching in Perfect and Imperfect Worlds, Review of Economic Studies, 69, 925-942.

[14] _______ (2002b): Assortative Matching in a Nontransferable World, CEPR DP 3469, www.cepr.org/pubs/dps/DP3469.asp.

[15] ______ (2006a): Notes on Assortative Matching under Strict NTU, Unpublished Manuscript, http://homepages.ulb.ac.be/ plegros/documents/strict.pdf.

[16] (2006b): Notes on Assortative Matching in Two-Sided Continuum Economies, Unpublished Manuscript,

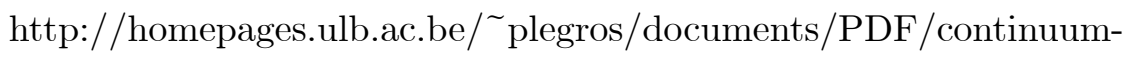
gid_implies_pam3.pdf.

[17] Milgrom, P., and C. Shannon (1994): Monotone Comparative Statics, Econometrica, 62, 157-180.

[18] Newman, A.F. (1999): Risk Bearing, Entrepreneurship, and the Theory of Moral Hazard, Unpublished Manuscript, http://www.econ.ucl.ac.uk/downloads/newman/risk.pdf.

[19] Prendergast, C. (2002): The Tenuous Trade-off between Risk and Incentives, Journal of Political Economy, 110, 1071-1102. 
[20] Roth, A., and M. Sotomayor (1990): Two-Sided Matching, Cambridge, England: Cambridge University Press.

[21] Rosenzweig, M.R., and O. Stark (1989): Consumption Smoothing, Migration, and Marriage: Evidence from Rural India, Journal of Political Economy, 97, 905-926.

[22] Serfes, K. (2005): Risk Sharing vs. Incentives: Contract Design under Two-Sided Heterogeneity, Economics Letters, 88, 343-349.

[23] Topkis, D.M. (1998): Supermodularity and Complementarity, Princeton, New Jersey: Princeton University Press.

[24] Wright, D.J. (2004): The Risk and Incentives Trade-off in the Presence of Heterogeneous Managers, Journal of Economics, 83, 209-223. 